\title{
Equivalent circuit models of two-layer flexure beams with excitation by temperature, humidity, pressure, piezoelectric or piezomagnetic interactions
}

\author{
U. Marschner ${ }^{1}$, G. Gerlach ${ }^{2}$, E. Starke ${ }^{3}$, and A. Lenk ${ }^{4, *}$ \\ ${ }^{1}$ Institute of Semiconductors and Microsystems, Technische Universität Dresden, 01062 Dresden, Germany \\ ${ }^{2}$ Institute of Solid-State Electronics, Technische Universität Dresden, 01062 Dresden, Germany \\ ${ }^{3}$ Institute of Lightweight Engineering and Polymer Technology, \\ Technische Universität Dresden, 01062 Dresden, Germany \\ ${ }^{4}$ Institute of Acoustics and Speech Communication, Technische Universität Dresden, 01062 Dresden, Germany \\ *professor emeritus
}

Correspondence to: U. Marschner (uwe.marschner@tu-dresden.de)

Received: 17 March 2014 - Revised: 9 July 2014 - Accepted: 16 July 2014 - Published: 17 September 2014

\begin{abstract}
Two-layer flexure beams often serve as basic transducers in actuators and sensors. In this paper a generalized description of their stimuli-influenced mechanical behavior is derived. For small deflection angles this description includes a multi-port circuit or network representation with lumped elements for a beam part of finite length. A number of coupled finite beam parts model the dynamic behavior including the first natural frequencies of the beam. For piezoelectric and piezomagnetic interactions, reversible transducer models are developed. The piezomagnetic two-layer beam model is extended to include solenoid and planar coils. Linear network theory is applied in order to determine network parameters and to simplify the circuit representation. The resulting circuit model is the basis for a fast simulation of the dynamic system behavior with advanced circuit simulators and, thus, the optimization of the system. It is also a useful tool for understanding and explaining this multi-domain system through basic principles of general system theory.
\end{abstract}

\section{Introduction}

Two-layer flexure beams have been present in engineering for more than 200 years. In the year 1766, a thermal bimetal strip was used for the first time in a practical application when it compensated environmental temperature influences in chronometers (Kašpar, 1960). Since then, a variety of two-layer problems that show the same effect have become known, but their operation is based on various physical causes (Fig. 1). In contrast with volume transducers, twolayer beams achieve significantly larger displacements, typically at the expense of a reduction in blocked force output. The mechanical transduction between deflection and stress is an essential property for sensor and energy-harvesting applications of two-layer beams, too. All these beams are described in the following in a unified representation as partially discussed by Gerlach and Lenk (1985). The large spectrum of technically possible realizations is limited to the problem of the plate strip.

The paper is organized as follows. In Sect. 2 the definition of the modeling of a unified one-dimensional two-layer beam element is narrowed down. The model is based on the material behavior state equations (Sect. 3) and ideal boundary conditions. Section 4 describes a differential beam element without shear forces and external pressure. From the beam element the general differential equation system of the actuating two-layer beam element for large angles and the differential equation system of the two-layer beam element for small angles are derived in Sects. 5 and 6, respectively. The latter is interpreted in Sect. 7 as a linear equivalent circuit of actuating two-layer beam elements, which is the main focus of the publication. The circuit description is extended in Sect. 8 to the compact low-frequency linear equivalent circuit of actuating two-layer beams. A number of coupled 


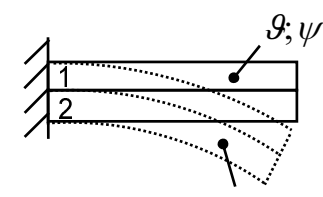

a) $\vartheta+\Delta \vartheta ; \psi+\Delta \psi$

b)
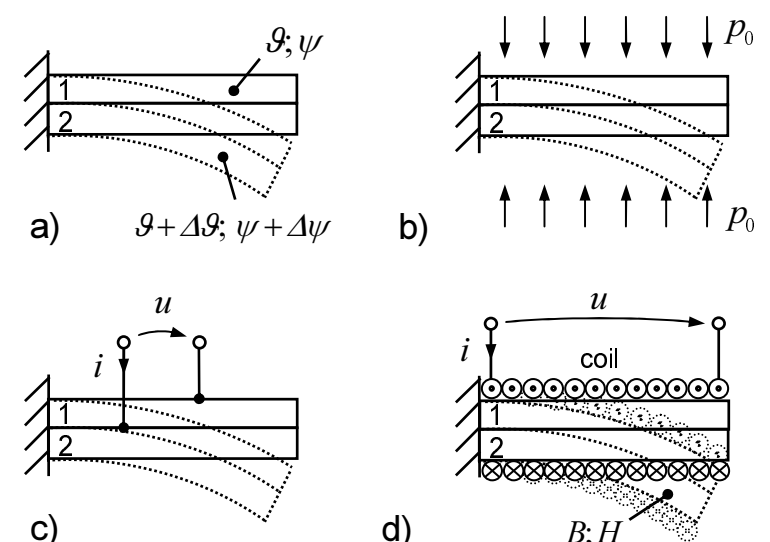

c)

d)

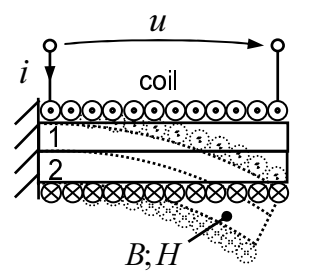

Figure 1. Two-layer flexure beams with excitation by (a) temperature $\vartheta$ or humidity $\Psi$, (b) pressure $p_{0}$, (c) piezoelectric and (d) piezomagnetic interactions.

finite beam elements model the dynamic behavior including the first natural frequency of a beam in Sect. 9 as a demonstration of a high-frequency linear equivalent circuit of an actuating two-layer beam. For piezoelectric and piezomagnetic interactions multi-port models of reversible transducers are developed in Sect. 10. This includes both piezoelectric and piezomagnetic unimorph transducers. Linear network theory is applied in order to determine network parameters and to simplify the circuit representation. Such a graphical representation of the system supports the understanding of the involved physical phenomena. Furthermore, it enables the usage of powerful circuit simulators to calculate the dynamic behavior of the system efficiently.

\section{Model of a unified one-dimensional two-layer beam element}

In the following, a one-dimensional two-layer beam element is part of a plate strip or beam. It consists of two homogeneous layers with different elastomechanical properties (Fig. 2). The influence quantity $\Lambda$, e.g., temperature, humidity etc., which is constant with respect to the space coordinates, induces a strain in the beam element.

At the beam sections oriented in direction $x_{1}$, a given distributed stress $T_{1}\left(x_{3}\right)$ and a transverse force $F_{3}$ act. The task is to determine the deflections $\xi_{1}\left(x_{1}\right)$ and $\xi_{3}\left(x_{1}\right)$, depending on the load pressures and forces incorporating the boundary conditions.

In order to solve the problem the following assumptions are made:

- The bond layer between the laminae is infinitesimally small and there is no flaw or gap in the bond layer. There is no shear deformation in the bond layer, i.e., the laminae cannot slip relative to each other.
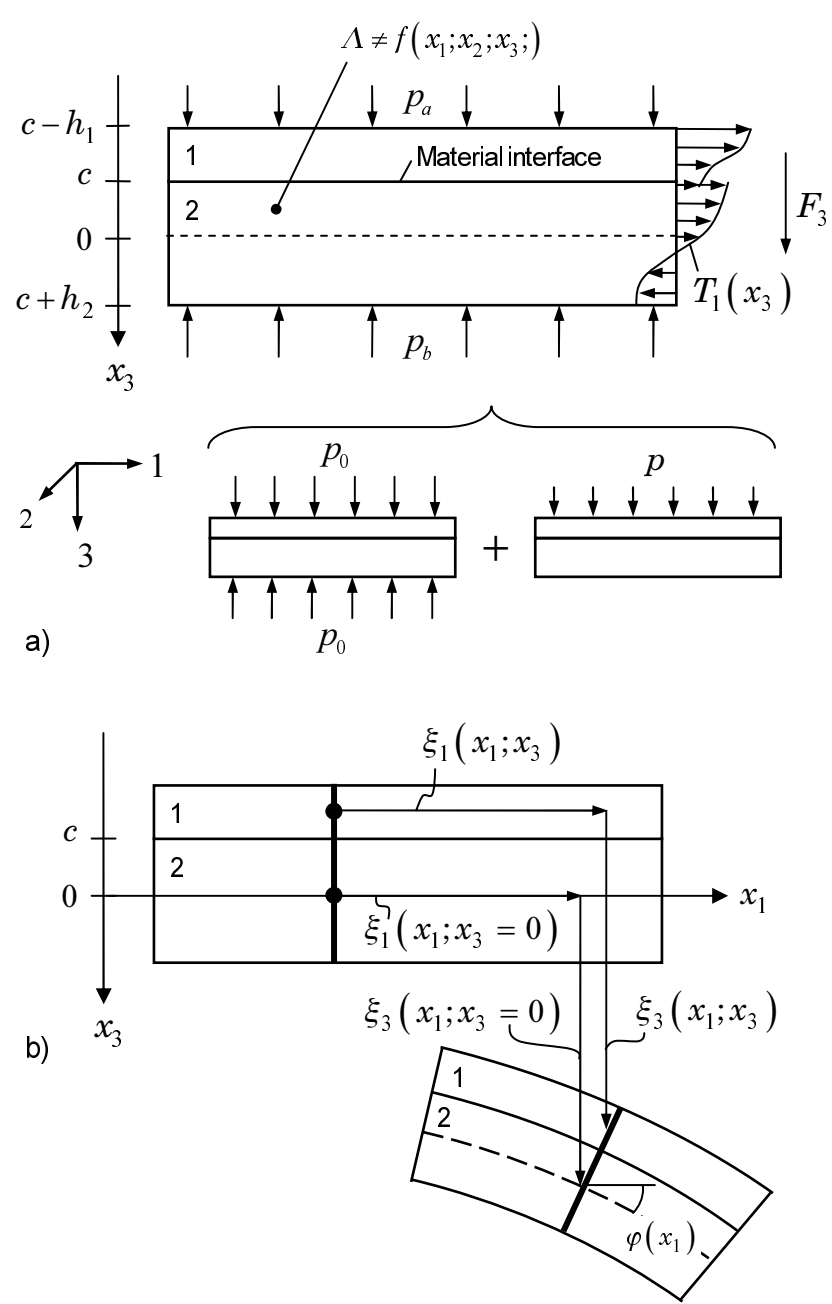

Figure 2. (a) Dynamic and (b) kinematic conditions at the twolayer beam element, where $\Lambda$ is the influence quantity; $x_{1}, x_{2}, x_{3}$ are coordinates; $\xi_{1}, \xi_{3}$ are deflections; $h_{1}, h_{2}$ are the thicknesses of the layers 1 and 2, respectively.; $c$ is the location of the neutral axis; $p, p_{0}, p_{a}=p_{0}+p, p_{b}=p_{0}$ are pressures; $F_{3}$ is a force; and $T_{1}$ is the normal stress.

- The bond layer has infinite stiffness and hence the composite beam behaves like a single-material beam element with integrated properties.

- Ideal elastic material behavior, i.e., validity of Hooke's law.

- According to Bernoulli's hypothesis, the structure is rigidly stiff. Rotational inertia is neglected and cross sections, originally perpendicular to the neutral plane or zero line, remain planar during deformation. In conjunction with Saint-Venant's principle, it is assumed that, after a short distance $\Delta x_{1}$, a distributed stress at the boundary $T_{1}\left(x_{3}\right)$ changes into a stress which is determined by a displacement function. Mean and moment 
of this displacement function are determined by the related quantities of the boundary distribution.

- The stress in $x_{3}$ direction is negligible $\left(T_{3}=0\right)$.

- Contributions of shear deformations to the displacement $\xi_{3}\left(x_{1}\right)$ can be neglected, i.e., the shear strains yield $S_{4}=$ $S_{5}=S_{6}=0$.

- The coordinate origin $x_{3}=0$ is placed at the distance $c$ from the bond layer. In this position the translational system points are located at the beam ends.

- Deflections of these system points at $x_{3}=0$ are labeled either with $\xi_{1}$ and $\xi_{3}$ or $\xi_{1}\left(x_{1}\right)$ and $\xi_{3}\left(x_{1}\right)$; deflections of arbitrary positions within the beam are labeled with $\left(\xi_{1}\left(x_{1} ; x_{3}\right), \xi_{3}\left(x_{1} ; x_{3}\right)\right)$

\section{Isotropic material behavior state equations}

All following considerations assume that isotropic material behavior can be described by the following state equations:

$S_{1}=\frac{1}{E_{0}} T_{1}-\frac{\nu_{0}}{E_{0}} T_{2}-\frac{\nu_{0}}{E_{0}} T_{3}+\alpha_{0} \Lambda$,

$S_{2}=-\frac{\nu_{0}}{E_{0}} T_{1}+\frac{1}{E_{0}} T_{2}-\frac{\nu_{0}}{E_{0}} T_{3}+\alpha_{0} \Lambda$,

$S_{3}=-\frac{\nu_{0}}{E_{0}} T_{1}-\frac{\nu_{0}}{E_{0}} T_{2}+\frac{1}{E_{0}} T_{3}+\alpha_{0} \Lambda$.

Reversibility is not considered at this stage but only actuation behavior. In order to be consistent with one-dimensional bending theory, $S_{2}$ and $S_{3}$ and $T_{2}$ and $T_{3}$ are defined by boundary conditions or viewed as strain-induced disturbance variables.

In the simplest case of a very small beam element without constant pressure $p_{0}$ (Fig. 3a), no stress components in $x_{2}$ and $x_{3}$ direction occur $\left(T_{2}=T_{3}=0\right)$ and Eq. (1a) simplifies to

$S_{1}=\frac{1}{E_{0}} T_{1}+\alpha_{0} \Lambda$.

In the case of a very wide beam, which is fixed at both ends $(w \gg l)$ as depicted in Fig. 3b, the boundary condition $S_{2}=0$ is acting internally. When, in addition, no constant pressure $p_{0}$ acts, for the stress $T_{3}=0$ follows, and for Eq. (1a) it yields

$S_{1}=\frac{1-v_{0}^{2}}{E_{0}} T_{1}+\left(1+v_{0}\right) \alpha_{0} \Lambda$.

In some micro-mechanical constructions the beam ends are not blocked as depicted in Fig. 3b, but the influence quantity $\Lambda$ loads the very wide beam element with a strain

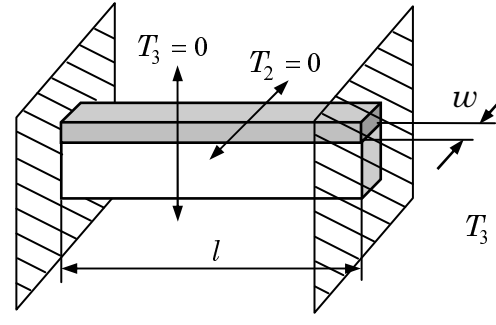

a) $p_{0}=0 ; l \gg w$

b)

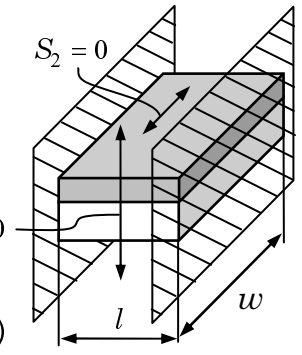

$p_{0}=0 ; w \gg l$

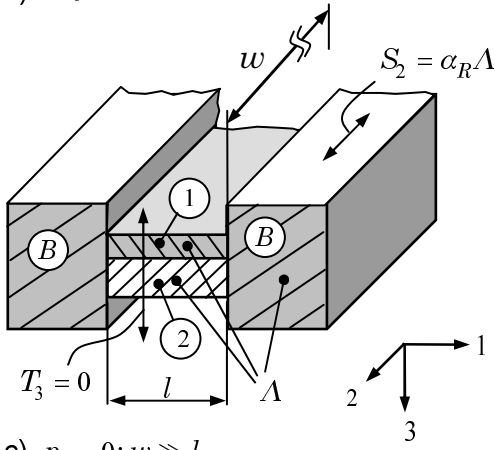

c) $p_{0}=0 ; w \gg l$

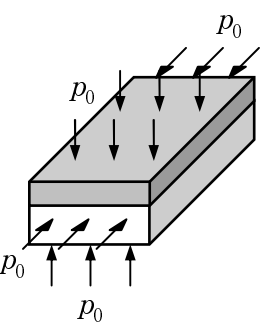

d) $T_{2}=T_{3}=-p_{0}$

Figure 3. Ideal boundary conditions at the beam.

$S_{2}=\alpha_{\mathrm{R}} \Lambda$ via influence coefficient $\alpha_{\mathrm{R}}$ (Fig. 3c). With $p_{0}=0$ Eq. (1a) here yields

$S_{1}=\frac{1-v_{0}^{2}}{E_{0}} T_{1}+\left[\left(1+v_{0}\right) \alpha_{0}-v_{0} \alpha_{\mathrm{R}}\right] \Lambda$.

In the special case of $\alpha_{R}=\alpha_{0}$, i.e., that the ends consist of one of the layers, Eq. (1a) simplifies further to

$S_{1}=\frac{1-v_{0}^{2}}{E_{0}} T_{1}+\alpha_{0} \Lambda$.

In the case of a constant pressure $p_{0}$, which also acts on the surfaces perpendicular to axis $x_{3}, T_{2}=T_{3}=-p_{0}$ yields conditions such that Eq. (1a) simplifies further to

$S_{1}=\frac{1}{E_{0}} T_{1}+\frac{2 v_{0}}{E_{0}} p_{0}$.

All considered cases can be described by the unified linear relation

$S_{1}=\frac{1}{E} T_{1}+\alpha \Lambda$.

The meaning of $1 / E$ and $\alpha$ in each case is summarized in Table 1. Even in the case of anisotropic material properties, generalized Eq. (7) applies.

\section{Differential beam element without shear forces and external pressure}

\subsection{Stresses and strains}

The object to be studied next is an arbitrary differential two-layer beam element of the plate strip. The layers are 
Table 1. Uniaxial stress conditions for real isotropic bimorph layers.

\begin{tabular}{|c|c|c|c|c|}
\hline \multicolumn{2}{|c|}{$\begin{array}{l}\text { Boundary condition } \\
\text { in direction }\end{array}$} & \multicolumn{2}{|c|}{$S_{1}=\frac{1}{E} T_{1}+\alpha \Lambda$} & \multirow{2}{*}{$\begin{array}{l}\text { see } \\
\text { Fig. }\end{array}$} \\
\hline$x_{3}$ & $x_{2}$ & $E$ & $\alpha$ & \\
\hline \multirow{4}{*}{$T_{3}=0$} & $T_{2}=0$ & $E_{0}$ & $\alpha_{0}$ & $3 a$ \\
\hline & $S_{2}=0$ & \multirow{3}{*}{$\frac{E_{0}}{1-v_{0}^{2}}$} & $\left(1+v_{0}\right) \alpha_{0}$ & $3 b$ \\
\hline & $S_{2}=\alpha_{R} \Lambda$ & & $\left(1+v_{0}\right) \alpha_{0}-v_{0} \alpha_{R}$ & \multirow{2}{*}{$3 \mathrm{c}$} \\
\hline & $S_{2}=\alpha_{0} \Lambda$ & & $\alpha_{0}$ & \\
\hline$T_{3}=-p_{0}$ & $T_{2}=-p_{0}$ & $E_{0}$ & $2 v_{0} / E_{0}$ & $3 d$ \\
\hline
\end{tabular}

characterized by their material properties $E_{1}, \alpha_{1}$ and $E_{2}, \alpha_{2}$ as well as their thicknesses $h_{1}$ and $h_{2}$. From Bernoulli's hypothesis follows that the deflection $\xi_{1}\left(x_{1} ; x_{3}\right)$ at a defined position is a linear function of coordinate $x_{3}$ :

$\xi_{1}\left(x_{1} ; x_{3}\right)=C_{a}\left(x_{1}\right) \cdot x_{3}+C_{b}\left(x_{1}\right)$.

The same conclusion applies to the strain:

$S_{1}\left(x_{1} ; x_{3}\right)=\frac{\mathrm{d} \xi_{1}}{\mathrm{~d} x_{1}}=\frac{\mathrm{d} C_{a}}{\mathrm{~d} x_{1}} \cdot x_{3}+\frac{\mathrm{d} C_{b}}{\mathrm{~d} x_{1}}$.

The differential beam element in Fig. 4, which is bent about the angle $\varphi\left(x_{1}\right)$, illustrates the meaning of $\mathrm{d} C_{a} / \mathrm{d} x_{1}$ and $\mathrm{d} C_{b} / \mathrm{d} x_{1}$ :

$S_{1}\left(x_{1} ; x_{3}\right)=\frac{\mathrm{d} \varphi}{\mathrm{d} x_{1}} \cdot x_{3}+S_{0}$,

where $S_{0}$ is the strain at $x_{3}=0$ :

$S_{0}=\left(\frac{\partial \xi_{1}\left(x_{1} ; x_{3}\right)}{\partial x_{1}}\right)_{x_{3}=0}=\frac{\Delta \xi_{1,0}}{\Delta x_{1}}=\frac{\mathrm{d} \xi_{1,0}}{\mathrm{~d} x_{1}}$.

Considering the material state equations which are valid in each region, the stress in the beam element yields

$T_{1}\left(x_{1} ; x_{3}\right)=\left\{\begin{array}{c}E_{1}\left(\frac{\mathrm{d} \varphi}{\mathrm{d} x_{1}} x_{3}+\frac{\mathrm{d} \xi_{1,0}}{\mathrm{~d} x_{1}}-\alpha_{1} \Lambda\right) \\ \text { for } c-h_{1} \leqslant x_{3} \leqslant c-0 \\ E_{2}\left(\frac{\mathrm{d} \varphi}{\mathrm{d} x_{1}} x_{3}+\frac{\mathrm{d} \xi_{1,0}}{\mathrm{~d} x_{1}}-\alpha_{2} \Lambda\right) \\ \text { for } c+0 \leqslant x_{3} \leqslant c+h_{2}\end{array}\right.$
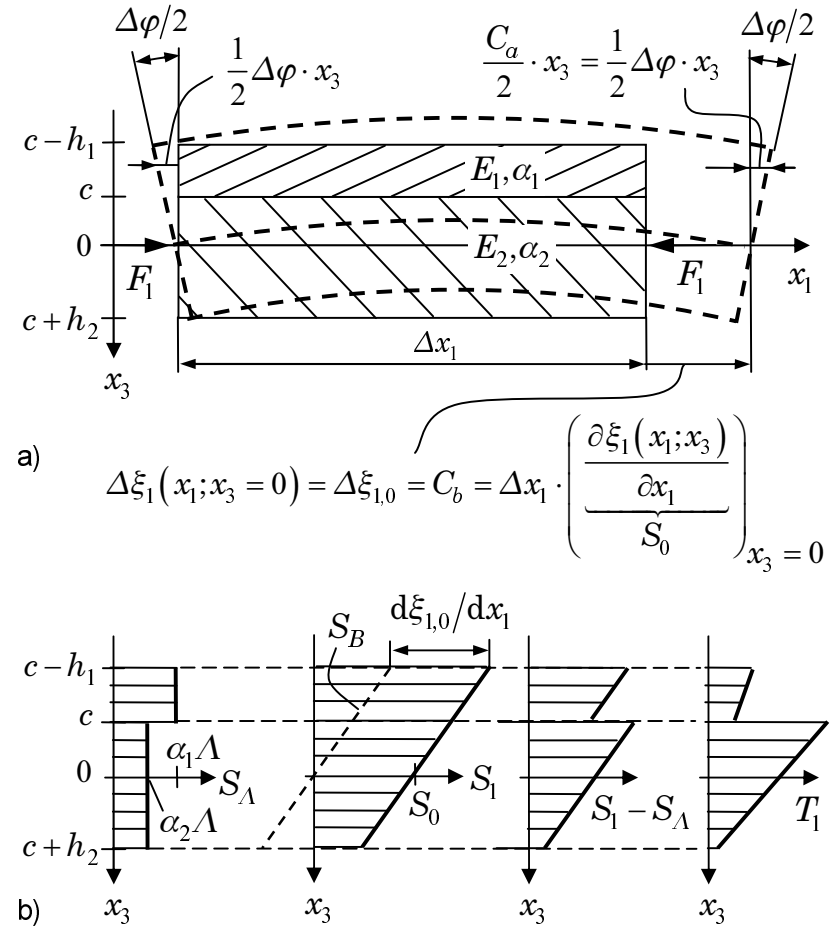

Figure 4. Strains and stresses in a two-layer beam: (a) bimorph element in non-deformed and deformed state; (b) strain and stress distributions at the cross section.

\subsection{Introduction of internal force $F_{1}$ and moment $M$ as coordinates}

From Eq. (12) the balancing normal force $F_{1}$ and balancing moment $M$ for the section follow with Fig. 5:

$$
\begin{aligned}
& F_{1}\left(x_{1}\right)=-w \int_{c-h_{1}}^{c+h_{2}} T_{1}\left(x_{3}\right) \mathrm{d} x_{3} \\
& =-w\left\{\left[-\frac{1}{2}\left(E_{1} h_{1}^{2}-E_{2} h_{2}^{2}\right)+\left(E_{1} h_{1}+E_{2} h_{2}\right) c\right] \frac{\mathrm{d} \varphi}{\mathrm{d} x_{1}}\right. \\
& \left.+\left(E_{1} h_{1}+E_{2} h_{2}\right) \frac{\mathrm{d} \xi_{1,0}}{\mathrm{~d} x_{1}}-\left(\alpha_{1} E_{1} h_{1}+\alpha_{2} E_{2} h_{2}\right) \Lambda\right\}, \\
& M\left(x_{1}\right)=-w \int_{c-h_{1}}^{c+h_{2}} T_{1}\left(x_{3}\right) \cdot x_{3} \mathrm{~d} x_{3} \\
& =-w\left\{\left[\frac{1}{3}\left(E_{1} h_{1}^{3}+E_{2} h_{2}^{3}\right)+\left(E_{1} h_{1}+E_{2} h_{2}\right) c^{2}\right.\right. \\
& \left.-\left(E_{1} h_{1}^{2}-E_{2} h_{2}^{2}\right) c\right] \frac{\mathrm{d} \varphi}{\mathrm{d} x_{1}} \\
& -\left[\frac{1}{2}\left(E_{1} h_{1}^{2}-E_{2} h_{2}^{2}\right)-\left(E_{1} h_{1}+E_{2} h_{2}\right) c\right] \frac{\mathrm{d} \xi_{1,0}}{\mathrm{~d} x_{1}} \\
& \left.-\Lambda\left[-\frac{1}{2}\left(\alpha_{1} E_{1} h_{1}^{2}-\alpha_{2} E_{2} h_{2}^{2}\right)+c\left(\alpha_{1} E_{1} h_{1}+\alpha_{2} E_{2} h_{2}\right)\right]\right\} .
\end{aligned}
$$


When $c$ is chosen at

$c=c_{S}=\frac{1}{2} \frac{E_{1} h_{1}^{2}-E_{2} h_{2}^{2}}{E_{1} h_{1}+E_{2} h_{2}}=\frac{\chi \zeta^{2}-1}{1+\chi \zeta} \cdot \frac{h_{2}}{2}$,

with $\chi=\frac{E_{1}}{E_{2}} \quad$ and $\quad \zeta=\frac{h_{1}}{h_{2}}$,

then $F_{1}$ does not depend on $\mathrm{d} \varphi / \mathrm{d} x_{1}$ nor $M$ on $\mathrm{d} \xi_{1} / \mathrm{d} x_{1}$. This distance from the material interface is the well-known location of the neutral layer in the case of an external moment. This case is in the following referred to as sensing case. With $c_{S}$, normal force and moment yield

$F_{1}\left(x_{1}\right)=\overline{E A}\left(\bar{\alpha} \Lambda-\frac{\mathrm{d} \xi_{1,0}}{\mathrm{~d} x_{1}}\right)$,

$M\left(x_{1}\right)=-\overline{E I} \frac{\mathrm{d} \varphi}{\mathrm{d} x_{1}}+M_{\Lambda}$,

with the equivalent linear expansion coefficient

$\bar{\alpha}=\frac{\alpha_{1} E_{1} h_{1}+\alpha_{2} E_{2} h_{2}}{E_{1} h_{1}+E_{2} h_{2}}$,

the length-related translational compliance or extensional stiffness of a homogeneous beam

$\overline{E A}=w\left(E_{1} h_{1}+E_{2} h_{2}\right)$,

the length-related rotational compliance or bending stiffness of a homogeneous beam

$$
\begin{aligned}
\overline{E I} & =\frac{w}{12} \frac{E_{1}^{2} h_{1}^{4}+E_{1} E_{2} h_{1} h_{2}\left(4 h_{1}^{2}+6 h_{1} h_{2}+4 h_{2}^{2}\right)+h_{2}^{4} E_{2}^{2}}{E_{1} h_{1}+E_{2} h_{2}} \\
& =\frac{w}{12} \frac{1+\chi^{2} \zeta^{4}+4 \chi \zeta^{3}+6 \chi \zeta^{2}+4 \chi \zeta}{\chi \zeta+1} \cdot h_{2}^{3} E_{2}
\end{aligned}
$$

and the moment source

$$
\begin{aligned}
M_{\Lambda} & =\frac{w}{2}\left[E_{1} \alpha_{1}\left(-h_{1}^{2}+2 c_{S} h_{1}\right)+E_{2} \alpha_{2}\left(h_{2}^{2}+2 c_{S} h_{2}\right)\right] \Lambda \\
& =-\frac{w}{2} \frac{E_{1} E_{2} h_{1} h_{2}\left(h_{1}+h_{2}\right)}{E_{1} h_{1}+E_{2} h_{2}}\left(\alpha_{1}-\alpha_{2}\right) \Lambda
\end{aligned}
$$

driven by $\Lambda$.

When a mean stress $\bar{T}_{1}=F_{1} / A$ is related to force $F_{1}$ then this stress determines the strain $S_{0}=\mathrm{d} \xi_{1,0} / \mathrm{d} x_{1}$. In addition, the difference $T_{1}\left(x_{1} ; x_{3}\right)-\bar{T}_{1}\left(x_{1}\right)=T_{B}\left(x_{1} ; x_{3}\right)$ represents the mean-free part of the stress distribution, whose moment determines the angle change $\mathrm{d} \varphi / \mathrm{d} x_{1}$ (Fig. 5).

\subsection{Curvature and neutral layer}

When there is no external force, i.e., $F_{1}=0$, it follows from Eq. (13) that the strain $\mathrm{d} \xi_{1,0} / \mathrm{d} x_{1}$ is given by

$$
\begin{aligned}
\frac{\mathrm{d} \xi_{1,0}}{\mathrm{~d} x_{1}} & =\left(\frac{1}{2} \frac{E_{1} h_{1}^{2}-E_{2} h_{2}^{2}}{E_{1} h_{1}+E_{2} h_{2}}-c\right) \frac{\mathrm{d} \varphi}{\mathrm{d} x_{1}} \\
& +\frac{\alpha_{1} E_{1} h_{1}+\alpha_{2} E_{2} h_{2}}{E_{1} h_{1}} \Lambda,
\end{aligned}
$$

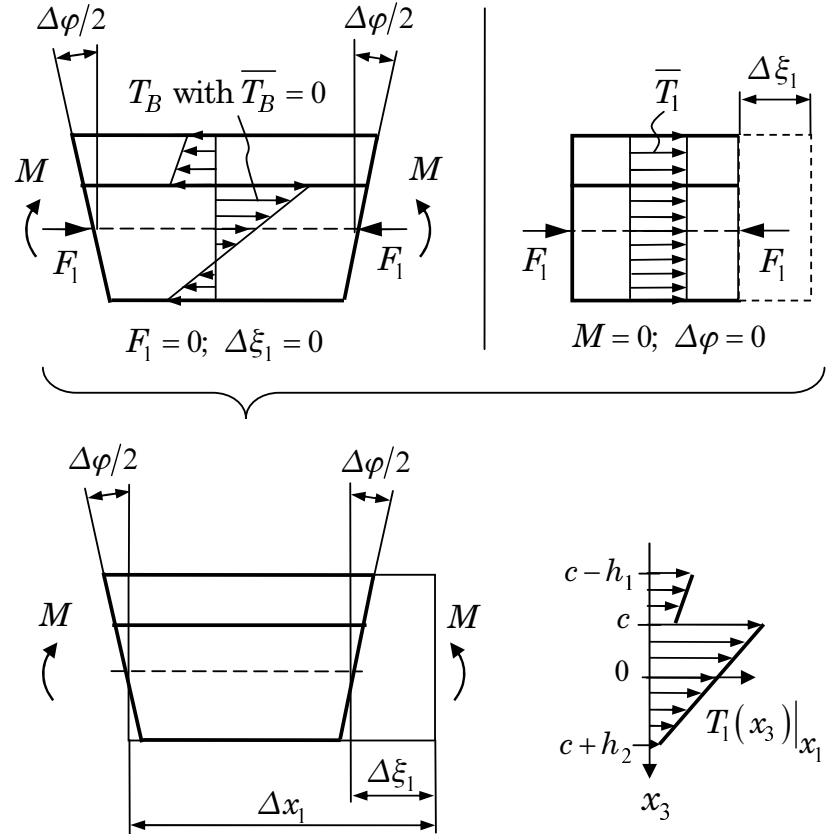

Figure 5. Forces and moments at the differential beam element without shear forces and difference pressure.

which is substituted in Eq. (14) to get the curvature

$$
\begin{aligned}
& \frac{\mathrm{d} \varphi}{\mathrm{d} x_{1}}=\frac{6 E_{1} h_{1} E_{2} h_{2}\left(h_{1}+h_{2}\right)\left(\alpha_{2}-\alpha_{1}\right) \Lambda}{h_{2}^{4} E_{2}^{2}+2 E_{2} E_{1} h_{2} h_{1}\left(2 h_{1}^{2}+3 h_{1} h_{2}+2 h_{2}^{2}\right)+E_{1}^{2} h_{1}^{4}} \\
& =\frac{6}{\left(h_{1}+h_{2}\right)} \cdot \frac{(1+\zeta)^{2}}{\frac{1}{\chi \zeta}+4\left(1+\zeta^{2}\right)+\chi \zeta^{3}+6 \zeta}\left(\alpha_{2}-\alpha_{1}\right) \Lambda
\end{aligned}
$$

as shown, e.g., by Guerrero and Wetherhold (2003) for magnetostrictive unimorphs. For a given total bimorph thickness $h=h_{1}+h_{2}$, the well-known maximum curvature results for $\chi=1 / \zeta^{2}$. Geometry and transduction coefficients have a larger effect on the deflection than a variation of Young's modulus (Gerlach and Dötzel, 2008).

Back substitution of Eq. (23) in Eq. (22) gives the location of the neutral layer in the actuation case:

$c_{A}=-\frac{h_{2}}{6} \frac{\left(4 \zeta^{2}+\frac{1}{\zeta \chi}\right) \mathcal{A}+3 \zeta(\mathcal{A}+1)+\zeta^{3} \chi+4}{(\zeta+1)(\mathcal{A}-1)}$,

with $\mathcal{A}=\frac{\alpha_{2}}{\alpha_{1}}$. The same location follows for the minimum of the potential energy of the deflected beam with regard to $c$ for $\mathrm{d} \xi_{1} / \mathrm{d} x_{1}=0$ :

$\frac{\mathrm{d}}{\mathrm{d} c}\left[\frac{1}{2} \int_{0}^{\Delta x_{1}} \frac{M_{\Lambda}^{2}}{\overline{E I}} \mathrm{~d} x_{1}\right]=0$ 
In the case of only layer 1 being active $\left(\alpha_{2}=0\right)$, Eq. (24) simplifies to

$$
\begin{aligned}
\left.c_{A}\right|_{\alpha_{2}=0} & =-\frac{1}{6} \frac{h_{1}^{3} E_{2}+3 E_{1} h_{1} h_{2}^{2}+4 E_{1} h_{2}^{3}}{h_{2} E_{1}\left(h_{1}+h_{2}\right)} \\
& =-\frac{h_{2}}{6} \frac{3 \zeta+4+\chi \zeta^{3}}{1+\zeta} .
\end{aligned}
$$

When the active layer is thin $\left(h_{1} \approx 0\right)$, the neutral layer is located at $-h_{2} \cdot 2 / 3$ and not at $-h_{2} / 2$, where it is located in the sensing case, i.e., for an external moment. This location matches that found by Stoney (1909) for thin films.

With the result in Eq. (24), the two-layer beam actually unveils pure bending $M \neq f\left(S_{0}\right)$ for two locations of the origin. Both locations, $c_{A}$ and $c_{S}$, give the same curvature for an induced stress. Figure 6 depicts $c$ normalized to the substrate thickness as a function of a given excitation $\alpha_{1} \Lambda$, normalized to the curvature. Therefore, the curvature or the deflection angle, respectively, can be calculated with $c_{S}$ countenancing $S_{0}$. Then the source moment can be treated as an external moment acting on the unimorph with the compliance $n_{\mathrm{R}}$ as a prerequisite for a reversible transducer model in Sect. 10.

\section{General differential equation system of the actuating two-layer beam element for large angles}

In addition to the previous considerations, the influence of a shear force $F_{3}$ and of a difference pressure $p$ on the twolayer beam element is now taken into account. In order to obtain a general differential equation system model of the two-layer beam element, the deformed element is considered in the transformed coordinate system $\left(x_{1}^{*}, x_{2}, x_{3}^{*}\right)$ according to Fig. 7, where $\left(x_{1}, x_{2}, x_{3}\right)$ serves as reference system for the deflections $\xi_{1}$ and $\xi_{3}$. This leads to the following relationships:

- As illustrated by Fig. 7b, the length of the deformed originally $\Delta x_{1}$-long - bimorph element becomes

$$
\begin{aligned}
\Delta x_{1} \cdot\left(1+S_{0}^{*}\left(x_{1}\right)\right) & =\Delta x_{1}+\Delta \xi_{1,0}^{*}\left(x_{1}\right) \\
& =\frac{1}{\cos \varphi}\left(\Delta x_{1}+\Delta \xi_{1,0}\left(x_{1}\right)\right) \\
\Delta x_{1} \cdot\left(1+S_{0}^{*}\left(x_{1}\right)\right) & =\frac{1}{\cos \varphi} \Delta x_{1} \cdot\left(1+S_{0}\left(x_{1}\right)\right) .
\end{aligned}
$$

For the strain $S_{0}\left(x_{1}\right)=\mathrm{d} \xi_{1,0} / \mathrm{d} x_{1}$,

$$
S_{0}\left(x_{1}\right)=\cos \varphi \cdot\left(1+S_{0}^{*}\left(x_{1}\right)\right)-1
$$

and for the relationship between the deflections $\xi_{1,0}\left(x_{1}\right)$ in the $x_{1}$ direction and $\xi_{1,0}^{*}\left(x_{1}\right)$ in the $x_{1}^{*}$ direction

$$
\frac{\mathrm{d} \xi_{1,0}}{\mathrm{~d} x_{1}}=\cos \varphi \cdot\left(1+\frac{\mathrm{d} \xi_{1,0}^{*}}{\mathrm{~d} x_{1}}\right)-1 .
$$

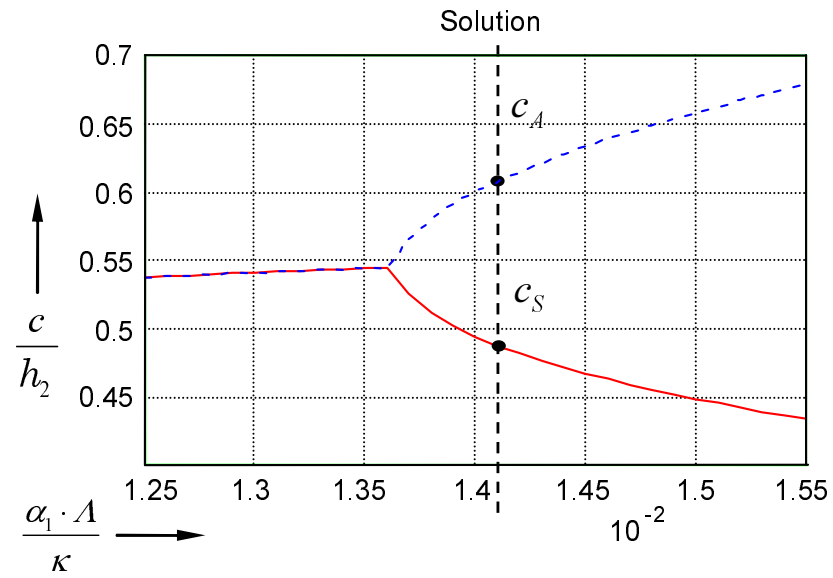

Figure 6. Solution of Eq. (14) with $\mathrm{d} \xi_{1,0} / \mathrm{d} x_{1}=0$ and $\alpha_{2}=0$ to the neutral-layer position $c$ normalized to $h_{2}$ exemplarily for $h_{1}=1 \mathrm{~mm}, E_{1}=23 \mathrm{GPa}, h_{2}=5 \mathrm{~mm}, E_{2}=71 \mathrm{GPa}, w=5 \mathrm{~mm}$, $l=0.1 \mathrm{~m}$ and $\alpha_{2}=0$.

- The force $F_{1}^{*}\left(x_{1}\right)$, which acts at the cross section of the element, consists of two components in the $x_{1}$ - and $x_{3}$ directions:

$$
F_{1}^{*}\left(x_{1}\right)=F_{1} \cos \varphi+F_{3} \sin \varphi .
$$

- A pressure $p$ acting on one side of the beam element causes two acting force components in the $x_{1}$ and $x_{3}$ directions, too (Fig. 7).

For the deformed beam element in coordinate system $\left(x_{1}^{*}, x_{2}, x_{3}^{*}\right)$, the following holds, which is consistent with Eqs. (16) and (17) from Sect. 4.2:

$$
F_{1}^{*}\left(x_{1}\right)=\overline{E A}\left(\bar{\alpha} \Lambda-\frac{\mathrm{d} \xi_{1,0}^{*}}{\mathrm{~d} x_{1}}\right),
$$

$M\left(x_{1}\right)=-\overline{E I} \frac{\mathrm{d} \varphi}{\mathrm{d} x_{1}}+M_{\Lambda}$.

The coefficients $\bar{\alpha}, \overline{E A}, \overline{E I}$ and $M_{\Lambda}$ are identical with the coefficients in Eqs. (16) and (17).

From Fig. 7 the following relations can also be derived:

- horizontal balance of forces:

$$
\begin{aligned}
& F_{1}\left(x_{1}+\Delta x_{1}\right)-F_{1}\left(x_{1}\right) \\
& +p \cdot w \cdot \Delta x_{1} \cdot\left(1+S_{0}^{*}\right) \cdot \sin \varphi=0 ;
\end{aligned}
$$

- vertical balance of forces:

$$
\begin{aligned}
& F_{3}\left(x_{1}+\Delta x_{1}\right)-F_{3}\left(x_{1}\right) \\
& -p \cdot w \cdot \Delta x_{1}\left(1+S_{0}^{*}\right) \cdot \cos \varphi=0 ;
\end{aligned}
$$




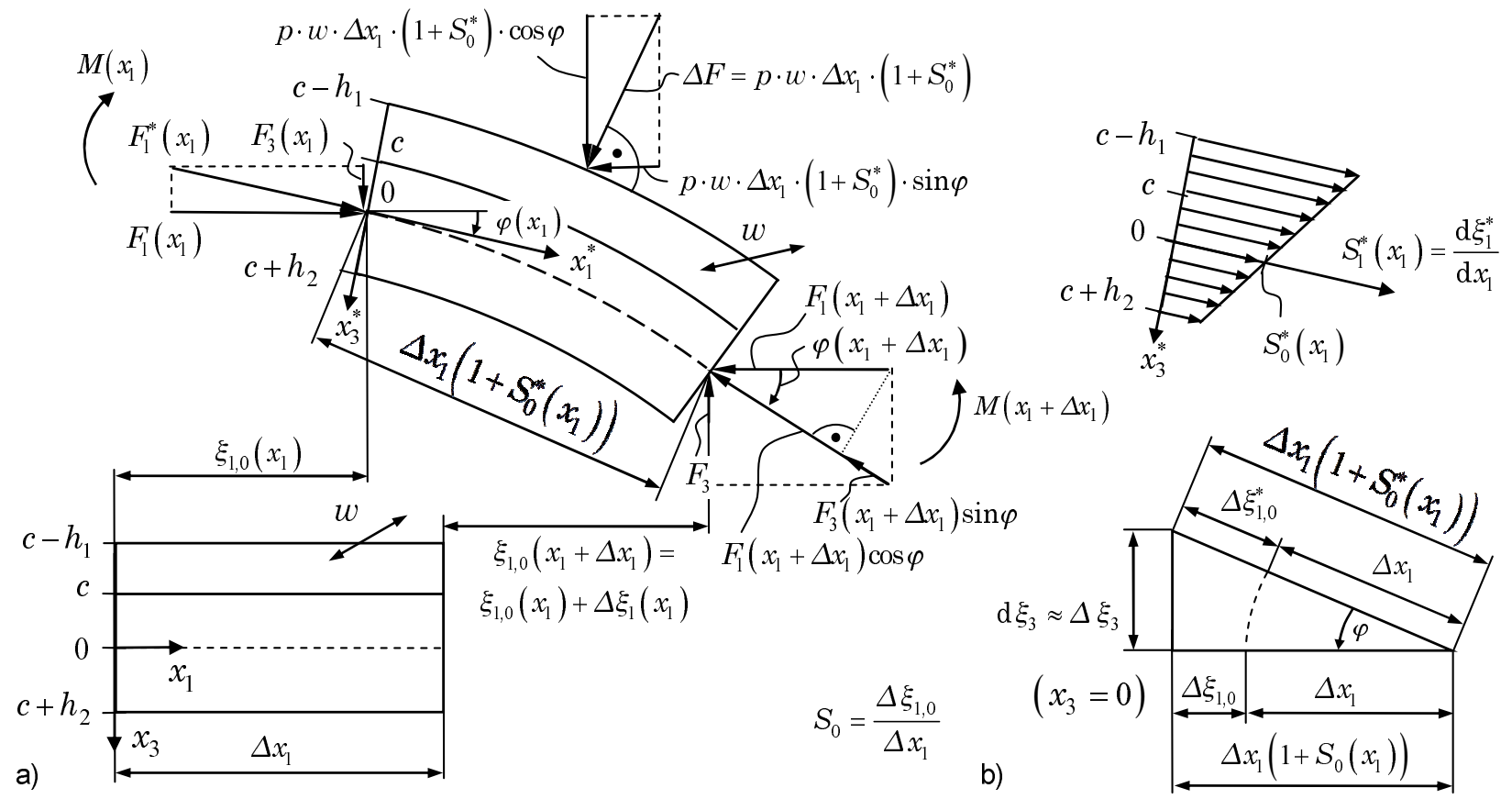

Figure 7. Definitions at the two-layer beam element with respect to large angles.

- balance of moment:

$$
\begin{aligned}
& M\left(x_{1}+\Delta x_{1}\right)-M\left(x_{1}\right) \\
& -F_{1}\left(x_{1}+\Delta x_{1}\right) \cdot \Delta x_{1} \cdot\left(1+S_{0}^{*}\right) \cdot \sin \varphi \\
& +F_{3}\left(x_{1}+\Delta x_{1}\right) \cdot \Delta x_{1} \cdot\left(1+S_{0}^{*}\right) \cdot \cos \varphi \\
& \approx p \cdot w \cdot \Delta x_{1} \cdot\left(1+S_{0}^{*}\right) \cdot \frac{\Delta x_{1} \cdot\left(1+S_{0}^{*}\right)}{2} \approx 0 ;
\end{aligned}
$$

- kinematics (Fig. 7b):

$$
\xi_{3}\left(x_{1}+\Delta x_{1}\right)-\xi_{3}\left(x_{1}\right)=\Delta x_{1}\left(1+S_{0}\right) \cdot \tan \varphi .
$$

Equations (33)-(36) together with Eqs. (27) and (32) constitute the differential equation set of a general plate strip element in set of equations (SOE) (37). Its validity is restricted by

- the assumption of small deflections $\mathrm{d}^{2} \xi_{1} / \mathrm{d} x_{1}^{2}$;

- the limited validity of Bernoulli's hypothesis;

- nonlinear stress-strain relations that occur in reality and

- the idealized formulation of the boundary conditions for $S_{2}$ and $T_{2}$.

$$
\begin{aligned}
\frac{\mathrm{d} F_{1}}{\mathrm{~d} x_{1}}= & -p \cdot w \cdot \sin \varphi \cdot[1+\bar{\alpha} \Lambda \\
& \left.-\frac{1}{\overline{E A}}\left(F_{1} \cos \varphi+F_{3} \sin \varphi\right)\right] \\
\frac{\mathrm{d} \xi_{1,0}}{\mathrm{~d} x_{1}}= & S_{0}=\cos \varphi \cdot[1+\bar{\alpha} \Lambda \\
& \left.-\frac{1}{\overline{E A}}\left(F_{1} \cos \varphi+F_{3} \sin \varphi\right)\right]-1 \\
\frac{\mathrm{d} F_{3}}{\mathrm{~d} x_{1}}= & p \cdot w \cdot \cos \varphi \cdot[1+\bar{\alpha} \Lambda \\
& \left.-\frac{1}{\overline{E A}}\left(F_{1} \cos \varphi+F_{3} \sin \varphi\right)\right] \\
\frac{\mathrm{d} \xi_{3}}{\mathrm{~d} x_{1}}= & \tan \varphi \cdot[1+\bar{\alpha} \Lambda \\
& \left.-\frac{1}{\overline{E A}}\left(F_{1} \cos \varphi+F_{3} \sin \varphi\right)\right] \\
\frac{\mathrm{d} M}{\mathrm{~d} x_{1}}= & \left(F_{1} \sin \varphi-F_{3} \cos \varphi\right) \cdot[1+\bar{\alpha} \Lambda \\
& \left.-\frac{1}{\overline{E A}}\left(F_{1} \cos \varphi+F_{3} \sin \varphi\right)\right] \\
\frac{\mathrm{d} \varphi}{\mathrm{d} x_{1}}= & -\frac{1}{\overline{E I}}\left(M+M_{\Lambda}\right)
\end{aligned}
$$

For $F_{1}\left(\xi_{1}\left(x_{1}\right)=0\right)>0$, i.e., if the beam is axially compressed, courses of the functions $F_{3}, \xi_{3}, M, \varphi=f\left(x_{1}\right)$ can result which do not fulfill the demand for biuniqueness. 
This problem must be solved with stability theory methods (Pflüger, 1975).

\section{Differential equation system of the two-layer beam element for small angles}

Contrary to the previous considerations, Fig. 8 depicts the definitions of the involved quantities and directions when small angles and deflections are assumed. With these definitions the following relations can be derived:

- balances of forces:

$$
\begin{aligned}
& F_{1}\left(x_{1}+\Delta x_{1}\right)-F_{1}\left(x_{1}\right)=0, \\
& F_{3}\left(x_{1}+\Delta x_{1}\right)-F_{3}\left(x_{1}\right)-p w \Delta x_{1}=0 ;
\end{aligned}
$$

- balance of moment:

$$
\begin{aligned}
& M\left(x_{1}+\Delta x_{1}\right)-M\left(x_{1}\right) \\
& +F_{1}\left(x_{1}+\Delta x_{1}\right) \cdot \Delta x_{1} \cdot \varphi\left(x_{1}\right) \\
& +F_{3}\left(x_{1}+\Delta x_{1}\right) \cdot \Delta x_{1}=p w \Delta x_{1} \cdot \frac{\Delta x_{1}}{2} \approx 0 ;
\end{aligned}
$$

- kinematics:

$$
\xi_{3}\left(x_{1}+\Delta x_{1}\right)-\xi_{3}\left(x_{1}\right)=\varphi\left(x_{1}\right) \cdot \Delta x_{1} .
$$

From Eqs. (16) to (41) SOE (42) can be derived. With this set of differential equations almost all problems can be solved where $\left|\varphi\left(x_{1}\right)\right| \ll 1$ and $F_{1} \leqslant 0$ hold. For the case $F_{1}\left(\xi_{1}\left(x_{1}\right)\right)>0$, the same remarks as in Sect. 5 apply.

$$
\begin{aligned}
& \frac{\mathrm{d} F_{1}}{\mathrm{~d} x_{1}}=0 \\
& \frac{\mathrm{d} \xi_{1,0}}{\mathrm{~d} x_{1}}=S_{0}=\bar{\alpha} \Lambda-\frac{1}{\overline{E A}} F_{1} \\
& \frac{\mathrm{d} F_{3}}{\mathrm{~d} x_{1}}=p \cdot w \\
& \frac{\mathrm{d} \xi_{3}}{\mathrm{~d} x_{1}}=\varphi \\
& \frac{\mathrm{d} M}{\mathrm{~d} x_{1}}=F_{1} \varphi-F_{3} \\
& \frac{\mathrm{d} \varphi}{\mathrm{d} x_{1}}=-\frac{1}{\overline{E I}}\left(M+M_{\Lambda}\right)
\end{aligned}
$$

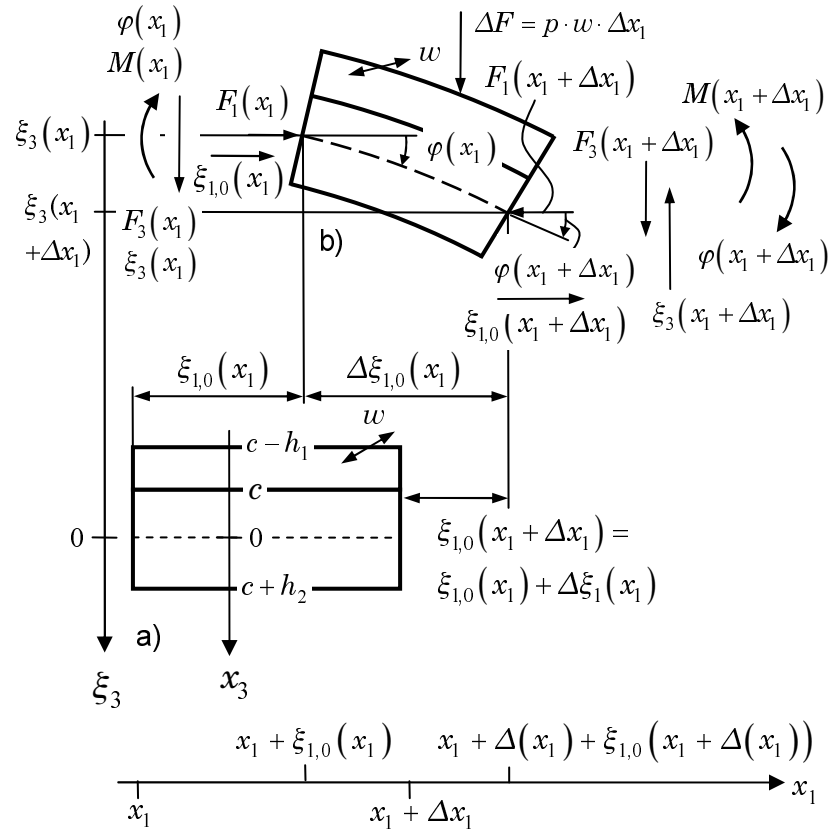

Figure 8. Definitions at the two-layer beam element for small angles: (a) non-deformed and (b) deformed bimorph.

\section{Linear equivalent circuits of actuating two-layer beam elements}

The differential equation system (42) is nonlinear due to the coupling $F_{1} \cdot \varphi$. The nonlinearity can be avoided in two ways:

1. For small forces $F_{1}$ the condition $\left|F_{1} \cdot \varphi\right| \ll F_{3}$ is assumed. This will change Eq. (42e) to

$$
\frac{\mathrm{d} M}{\mathrm{~d} x_{1}}=-F_{3} .
$$

2. The force $F_{1}$ is treated as a time- and motionindependent source quantity.

In order to obtain a circuit representation of the linearized set of Eq. (42) the time-dependent complex coordinates angular velocity

$\underline{\Omega}=\frac{\mathrm{d} \underline{\underline{d}}}{\mathrm{~d} t}=\mathrm{j} \omega \underline{\varphi}$

and velocity

$\underline{v}_{1 ; 3}=\frac{\mathrm{d} \underline{\xi}_{1,0 ; 3}}{\mathrm{~d} t}=\mathrm{j} \omega \underline{\xi}_{1 ; 3}$

are introduced. Here, $\omega$ is the angular frequency and $\mathrm{j}$ the imaginary unit. Integration of Eq. (42) over $x_{1}$ then gives the following solution:

$\underline{F}_{1}=\underline{F}_{1}\left(x_{1}\right)$, 
$\underline{v}_{1}=\mathrm{j} \omega \bar{\alpha} \underline{\Lambda} x_{1}-\mathrm{j} \omega \frac{x_{1}}{\overline{E A}} \underline{F}_{1}\left(x_{1}\right)+\underline{v}_{1}\left(x_{1}\right)$,

$\underline{F}_{3}=\underline{p} \cdot w \cdot x_{1}+\underline{F}_{3}\left(x_{1}\right)$,

$\underline{M}=\frac{F_{1} \Delta x_{1}}{\mathrm{j} \omega} \underline{\Omega}\left(x_{1}+\Delta x_{1}\right)-\underline{p} \cdot w \frac{x_{1}^{2}}{2}$

$$
-\underline{F}_{3}\left(x_{1}\right) x_{1}+\underline{M}\left(x_{1}\right),
$$

$$
\underline{\Omega}=\frac{\mathrm{j} \omega}{\overline{E I}}\left(\underline{p} \cdot w \frac{x_{1}^{3}}{6}+\underline{F}_{3}\left(x_{1}\right) \frac{x_{1}^{2}}{2}-\underline{M}\left(x_{1}\right) x_{1}\right.
$$$$
\left.-M_{\Lambda} x_{1}\right)+\underline{\Omega}\left(x_{1}\right),
$$

$$
\begin{aligned}
\underline{v}_{3} & =\frac{\mathrm{j} \omega}{\overline{E I}}\left(\underline{p} \cdot w \frac{x_{1}^{4}}{24}+\underline{F}_{3}\left(x_{1}\right) \frac{x_{1}^{3}}{6}-\underline{M}\left(x_{1}\right) \frac{x_{1}^{2}}{2}\right. \\
& \left.-M_{\Lambda} \frac{x_{1}^{2}}{2}\right)+\underline{\Omega}\left(x_{1}\right) x_{1}+\underline{v}_{3}\left(x_{1}\right) .
\end{aligned}
$$

Application of the boundary conditions at the left side of the differential bending element $\underline{F}_{1,3}\left(x_{1}\right), \underline{v}_{1,3}\left(x_{1}\right), \underline{M}\left(x_{1}\right)$ and $\underline{\Omega}\left(x_{1}\right)$ and at the right side $\underline{F}_{1,3}\left(x_{1}+\Delta x_{1}\right), \underline{v}_{1,3}\left(x_{1}+\Delta x_{1}\right)$, $\underline{M}\left(x_{1}+\Delta x_{1}\right)$ and $\underline{\Omega}\left(x_{1}+\Delta x_{1}\right)$ acting at a beam element of length $\Delta x_{1}$ and using the approximation of Eq. (40) yields the following:

$\circledast_{1}: \underline{F}_{1}\left(x_{1}+\Delta x_{1}\right)=\underline{F}_{1}\left(x_{1}\right)$,

$\mathcal{O}_{1}: \underline{v}_{1}\left(x_{1}+\Delta x_{1}\right)=\Delta \underline{v}_{\Lambda}-\mathrm{j} \omega \Delta n \cdot \underline{F}_{1}\left(x_{1}\right)+\underline{v}_{1}\left(x_{1}\right)$,

$\circledast_{2}: \underline{F}_{3}\left(x_{1}+\Delta x_{1}\right)=\Delta \underline{F}+\underline{F}_{3}\left(x_{1}\right)$,

$$
\begin{aligned}
\circledast_{3}: & \underline{M}\left(x_{1}+\Delta x_{1}\right)=\underline{M}\left(x_{1}\right)-\frac{\underline{\Omega}\left(x_{1}+\Delta x_{1}\right)}{\mathrm{j} \omega \Delta n_{F_{1}}} \\
& -\left(\Delta \underline{F}+\underline{F}_{3}\left(x_{1}\right)\right) \frac{\Delta x_{1}}{2}-\underline{F}_{3}\left(x_{1}\right) \frac{\Delta x_{1}}{2}, \\
\mathcal{O}_{2}: & \underline{\Omega}\left(x_{1}+\Delta x_{1}\right) \approx \underline{\Omega}\left(x_{1}\right)-\Delta \underline{\Omega}_{\Lambda}
\end{aligned}
$$$$
-\mathrm{j} \omega \Delta n_{\mathrm{R}} \cdot[\underline{M}\left(x_{1}\right)-\underline{F}_{3}\left(x_{1}\right) \frac{\Delta x_{1}}{2}+\underbrace{\Delta F \frac{\Delta x_{1}}{6}}_{\approx 0}],
$$

$$
\begin{aligned}
\mathcal{O}_{3}: \underline{v}_{3}\left(x_{1}+\Delta x_{1}\right) \approx \underline{v}_{3}\left(x_{1}\right)+\underline{\Omega}\left(x_{1}\right) \frac{\Delta x_{1}}{2} \\
+\underline{\Omega}\left(x_{1}+\Delta x_{1}\right) \frac{\Delta x_{1}}{2}-\underbrace{\mathrm{j} \omega \Delta n_{\mathrm{R}} \frac{\Delta x_{1}^{2}}{12} \cdot \underline{F}_{3}\left(x_{1}\right)}_{\approx 0},
\end{aligned}
$$

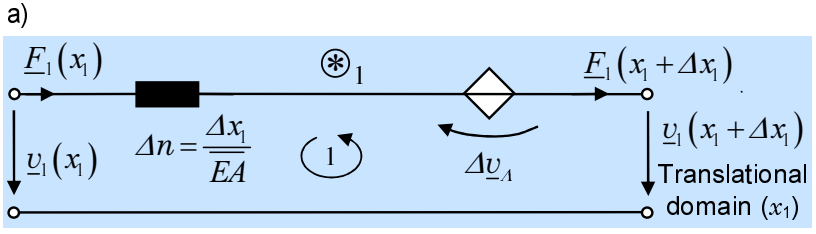

b)

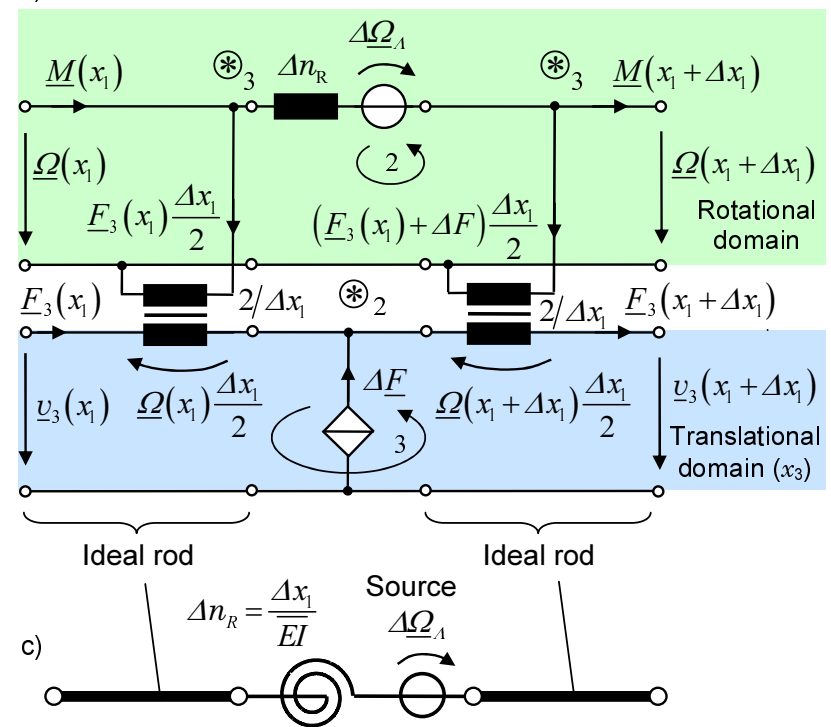

Bending compliance

Figure 9. Circuit representation of a two-layer beam element when $\left|F_{1} \cdot \varphi\right| \ll F_{3}$ in direction $x_{1}$ (a) and direction $x_{3}$ as well as of the dynamic rotational behavior (b), and system model with ideal rods as rotational-translational transducers (introduced by Schroth, 1968) (c). The axial velocity source $\Delta \underline{v}_{\Lambda}$ is related to the location $c_{S}$ $\left(\Delta \underline{v}_{\Lambda}=0\right.$ at the neutral axis $\left.c_{A}\right)$.

with

$$
\begin{aligned}
& \Delta \underline{v}_{\Lambda}=\mathrm{j} \omega \bar{\alpha} \underline{\Lambda} \Delta x_{1}, \\
& \Delta n=\frac{\Delta x_{1}}{\overline{E A}}, \\
& \Delta n_{F_{1}}=-\frac{1}{\left(F_{1} \Delta x_{1}\right)}, \\
& \Delta \underline{F}=\underline{p} \cdot w \cdot \Delta x_{1}, \\
& \Delta n_{\mathrm{R}}=\frac{\Delta x_{1}}{\overline{E I}}, \\
& \Delta \underline{\Omega}_{\Lambda}=\mathrm{j} \omega \Delta n_{\mathrm{R}} \cdot \underline{M}_{\Lambda} .
\end{aligned}
$$

Following Kirchhoff's laws, sums of flow quantities constitute nodes - marked with $\circledast$ and sums of across quantities constitute meshes - marked with 0 . Figures 9 and 10 depict the resulting circuit representation of the two-layer beam element for the two linearization cases of the first paragraph in Sect. 7. The angular velocity source $\Delta \underline{\Omega}_{\Lambda}$ can also be interpreted as moment source $\underline{M}_{\Lambda}$ acting on $\Delta n_{\mathrm{R}}$. For $\underline{\Lambda}=0$ and $h_{1}$ or $h_{2}=0$, these networks include the case of a homogeneous monomorph-plate strip which is subject to bending. 


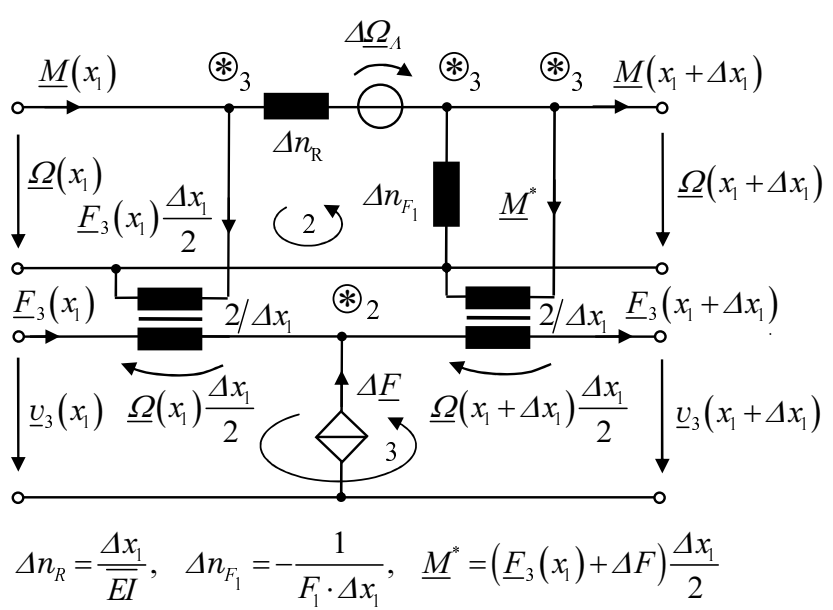

Figure 10. Circuit representation of a two-layer beam element when $F_{1} \neq F_{1}(t)$ (source quantity).

The approximations in Eqs. (47e) and (47f) do not apply for a large $\Delta x$. This case is treated in Sect. 8 .

All following equivalent circuits assume that $\left|F_{1} \cdot \varphi\right| \ll F_{3}$ and that no pressure load occurs.

\section{Low-frequency linear equivalent circuit of actuating two-layer beams}

In the previous section a beam element of finite length $\Delta x$ was derived. In order to describe a long beam of length $l$ for quasi-static processes without pressure load $(p=0)$ and with the assumption $\left|F_{1} \cdot \varphi\right| \ll F_{3}$, the beam elements in Fig. 9 can be connected in a chain-like manner. This chain circuit can be simplified to a much smaller four-port circuit as long as the links between the coordinates at the beam ends (I) and (II) are of interest. These coordinates become $\underline{F}_{\mathrm{I}}=\underline{F}_{3}(0), \underline{v}_{\mathrm{I}}=$ $\underline{v}_{3}(0), \underline{M}_{\mathrm{I}}=\underline{M}(0)$ and $\underline{\Omega}_{\mathrm{I}}=\underline{\Omega}(0)$ at the left side and $\underline{F}_{\mathrm{II}}=$ $\underline{F}_{3}(l), \underline{v}_{\mathrm{II}}=\underline{v}_{3}(l), \underline{M}_{\mathrm{II}}=\underline{M}(l)$ and $\underline{\Omega}_{\mathrm{II}}=\underline{\Omega}(l)$ at the right side, as depicted in Fig. 11. With $n_{0}=l^{3} / \overline{E I}$, integration of SOE (42) over beam length $l$ and application of the boundary conditions at (I) and (II) gives

$\underline{F}_{\mathrm{II}}=\underline{F}_{\mathrm{I}}$

$\underline{M}_{\mathrm{II}}=\underline{F}_{\mathrm{I}}-l \underline{M}_{\mathrm{I}}$,

$$
\begin{aligned}
\underline{\Omega}_{\mathrm{II}}= & -\mathrm{j} \omega \frac{n_{0}}{l^{2}}\left(\underline{M}_{\mathrm{I}}+\underline{M}_{\Lambda}\right)+\mathrm{j} \omega \frac{n_{0}}{2 l} \underline{F}_{\mathrm{I}}+\underline{\Omega}_{\mathrm{I}} \\
= & -\mathrm{j} \omega \frac{n_{0}}{2 l^{2}}(\underbrace{\underline{M}_{\mathrm{I}}-l \underline{F}_{\mathrm{I}}}_{\underline{M}_{\mathrm{II}}}+\underline{M}_{\Lambda}) \\
& \underbrace{-\mathrm{j} \omega \frac{n_{0}}{2 l^{2}}\left(\underline{M}_{\mathrm{I}}+\underline{M}_{\Lambda}\right)+\underline{\Omega}_{\mathrm{I}}}_{\underline{\Omega}_{T}},
\end{aligned}
$$

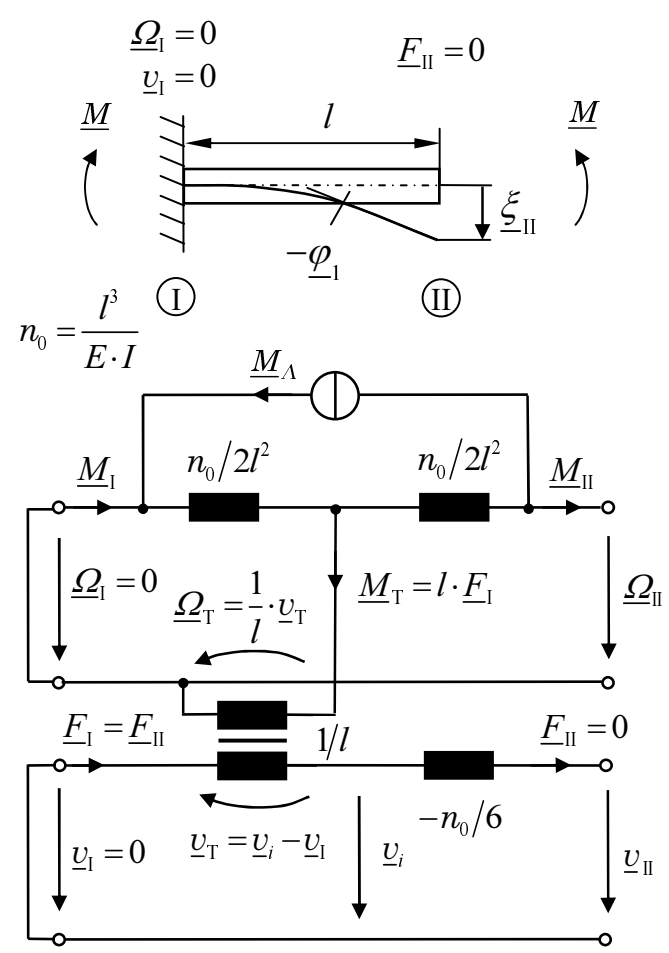

Figure 11. Low-frequency linear equivalent circuit of an actuating fixed-free two-layer beam without direction 1 .

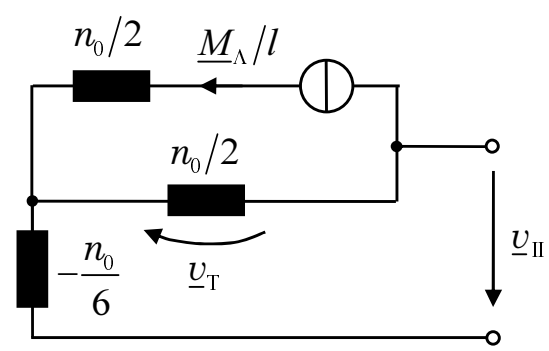

Figure 12. Translational linear equivalent circuit of an actuating fixed-free two-layer beam.

$$
\begin{aligned}
\underline{v}_{\mathrm{II}} & =-\mathrm{j} \omega \frac{n_{0}}{2 l}\left(\underline{M}_{\mathrm{I}}+\underline{M}_{\Lambda}\right)+\mathrm{j} \omega \frac{n_{0}}{6} \underline{F}_{\mathrm{I}}+l \underline{\Omega}_{\mathrm{I}}+\underline{v}_{\mathrm{I}} \\
& =\underbrace{l \cdot\left(\underline{\Omega}_{\mathrm{I}}-\underline{\Omega}_{T}\right)}_{\underline{v}_{T}}+\mathrm{j} \omega \frac{n_{0}}{6} \underline{F}_{\mathrm{I}}+\underline{v}_{\mathrm{I}} .
\end{aligned}
$$

The equivalent circuit is shown in Fig. 11 for the example of a fixed-free beam. The prevented movement and rotation at the left side are short-circuited across quantities. The translational domain includes the negative compliance $-n_{0} / 6$ which can be handled by circuit simulators. However, the total compliance of the bending beam is positive.

When only the translational behavior in direction 3 is of interest, then the network can be simplified. Figure 12 shows the result of the transformation of the rotational elements 

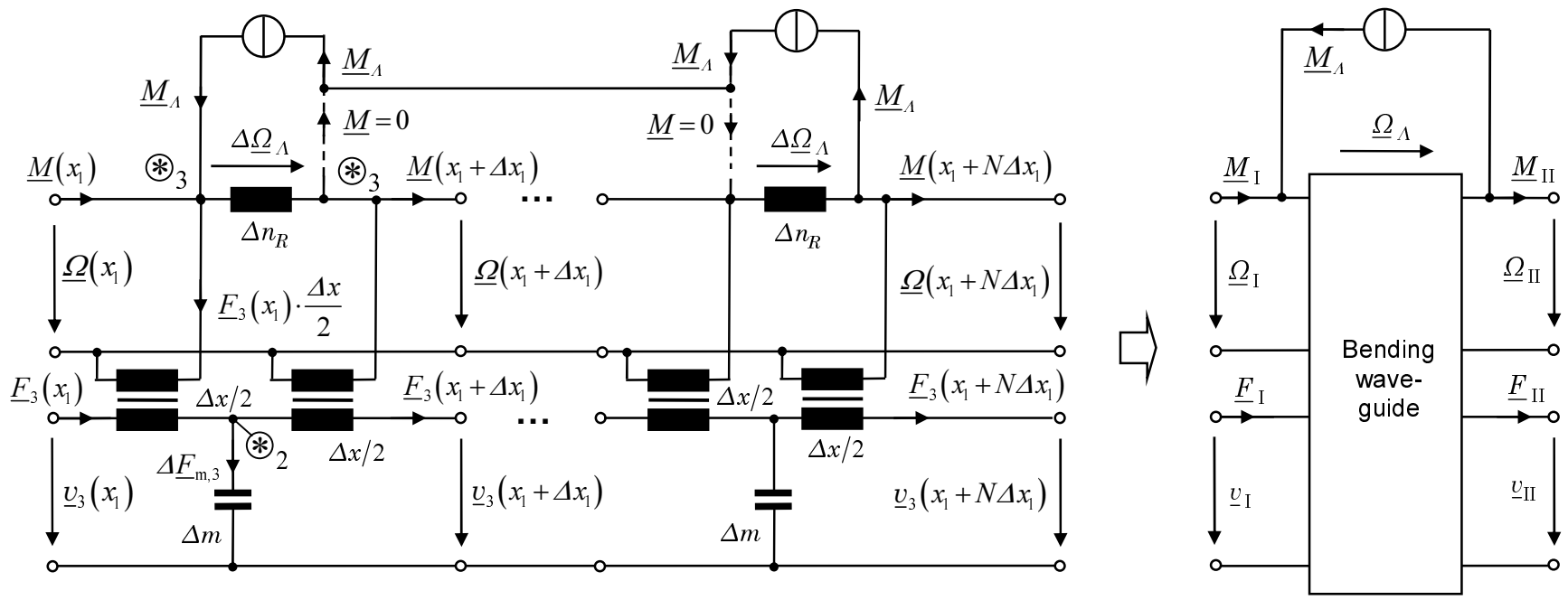

Figure 13. High-frequency linear equivalent circuit of direction 3 and dynamic rotational behavior of an actuating two-layer beam.

into the translational domain with $\underline{F}_{3}=\underline{M} / l$ and $n=n_{\mathrm{R}} \cdot l^{2}$. The force source linked to the transformed moment generates the velocity $\underline{v}_{W}$, which appears as $\underline{v}_{\mathrm{II}}$ when no force $\underline{F}_{\mathrm{II}}$ is present:

$\underline{v}_{T}=-\underline{v}_{\mathrm{II}}=-\mathrm{j} \omega \underline{\xi}_{\mathrm{II}}=-\mathrm{j} \omega \frac{n_{0}}{2} \frac{\underline{M}_{\Lambda}}{l}$.

With

$\underline{\Omega}_{\mathrm{II}}=\mathrm{j} \omega \underline{\varphi}_{\mathrm{II}}=-\frac{\mathrm{j} \omega n_{0}}{l^{2}} \cdot \underline{M}_{\Lambda}$,

it follows that

$\underline{\xi}_{\mathrm{II}}=-\frac{l}{2} \underline{\varphi}_{\mathrm{II}}$.

\section{High-frequency linear equivalent circuit of an actuating two-layer beam}

If no pressure difference is considered but the average mass is assumed for each beam element, then Eqs. (47a), (47c) and (47d) change in the case of $\left|F_{1} \cdot \varphi\right| \ll F_{3}$ to

$$
\begin{gathered}
\circledast_{1}: \underline{F}_{1}\left(x_{1}+\Delta x_{1}\right)=\underline{F}_{1}\left(x_{1}\right)+\Delta \underline{F}_{m, 1}, \\
\mathcal{O}_{1}: \underline{v}_{1}\left(x_{1}+\Delta x_{1}\right)=\Delta \underline{v}_{\Lambda}-\mathrm{j} \omega \Delta n \cdot \underline{F}_{1}+\underline{v}_{1}\left(x_{1}\right), \\
\circledast_{2}: \underline{F}_{3}\left(x_{1}+\Delta x_{1}\right)=\Delta \underline{F}_{m, 3}+\underline{F}_{3}\left(x_{1}\right), \\
\circledast_{3}: \underline{M}\left(x_{1}+\Delta x_{1}\right)=\underline{M}\left(x_{1}\right) \\
\quad-\left(\underline{F}_{m}+\underline{F}_{3}\left(x_{1}\right)\right) \frac{\Delta x_{1}}{2}-\underline{F}_{3}\left(x_{1}\right) \frac{\Delta x_{1}}{2},
\end{gathered}
$$

with

$$
\Delta \underline{F}_{m, 1}=\mathrm{j} \omega \Delta m \underline{v}_{1}\left(x_{1}+\Delta x_{1}\right)
$$

and

$\Delta \underline{F}_{m, 3}=\mathrm{j} \omega \Delta m \underline{v}_{3}\left(x_{1}+\Delta x_{1} / 2\right)$.

The beam element describing direction 3 includes its mass instead of the pressure-related force source in Fig. 9. Figure 13 shows the resulting mechanical network with distributed parameters without direction 1 when the beam is discretized over $x_{3}$ into $N$ finite beam elements. A finite beam element in direction 1 is represented by a circuit similar to Fig. 9a with the difference of the included mass $\Delta m$, which is connected to the mechanical reference (ground).

A thought experiment leads to the conclusion that the source moment is coupled at the beam ends and that it is not branched into the inner beam, i.e.,

$$
\Delta \underline{M}_{\Lambda}\left(\Delta x_{1}\right)=\underline{M}_{\Lambda}(l) .
$$

Therefore, the unimorph can be grouped into a bending wave guide with the moment source as an additional boundary condition, as shown in Fig. 13.

The structures constitute a longitudinal wave guide in direction 1 and the bending wave guide in Fig. 13. The behavior of this network can be investigated with standard circuit simulators, like SPICE (Simulation Program with Integrated Circuit Emphasis). An example is presented in Sect. 10.2.3. When the elements are connected in a chain-like manner then two connected rotational-translational transducers with a length of $\Delta x_{1} / 2$ can be combined to one single transducer with a length of $\Delta x_{1}$ as demonstrated in Fig. 32 .

\section{Multi-port models of reversible transducers}

Equation (7) describes the relation between the mechanical field pair $(S, T)$ and a transduction relation between the two physical domains. Reversibility is entailed by a second set of 


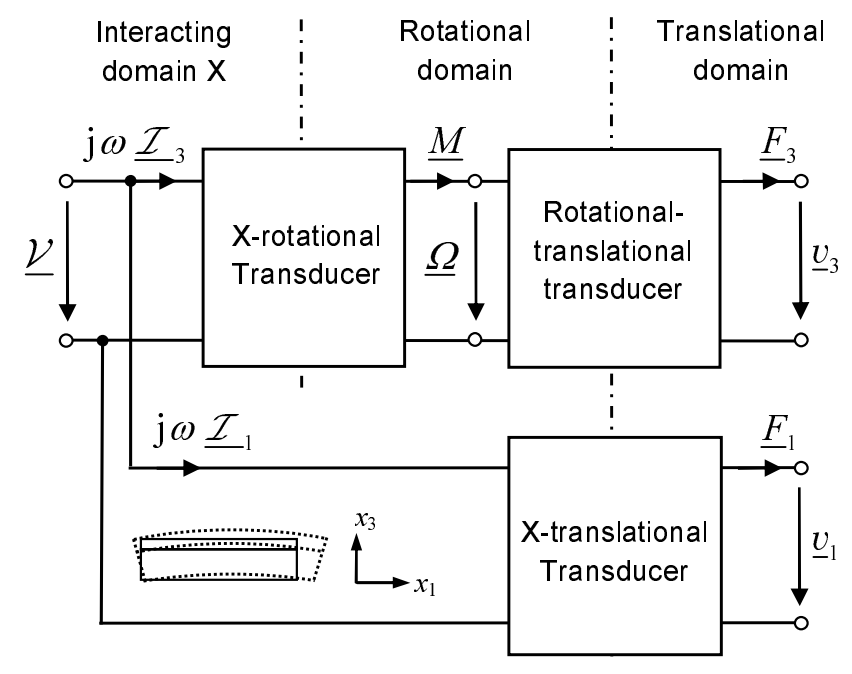

Figure 14. Across and flow coordinates in the circuit description involving the interacting domain $X$.

material state equations, which relates field pair components $\left(\Lambda_{i}, \mathcal{K}_{i}\right)$ of the interacting domain $X$ to each other in addition to the transduction mechanism. From this second set only the relation between the influence quantity $\underline{\Lambda}$, stress $\underline{T}_{1}$ or strain $\underline{S}_{1}$, obtained with Eq. (7), and quantity $\underline{\mathcal{K}}$ is considered:

$\underline{\mathcal{K}}=\alpha \underline{T}_{1}+\beta^{T} \underline{\Lambda}=\alpha E \underline{S}_{1}+\underbrace{\left(\beta^{T}-\alpha^{2} E\right)}_{\beta^{S}} \underline{\Lambda}$,

where $\beta$ is an additional constant material coefficient. With this second set a multi-domain port description including the physical domain $X$ can be derived. This domain controls the moment source, velocity or rotational velocity source in the previously developed models. Figure 14 shows the extended port description. Each translational direction involves a separate transducer which includes these sources. In the interacting domain $X$, the flow rate $\mathrm{j} \omega \mathcal{I}$ and across quantity $\underline{\mathcal{V}}$ serve as network coordinates, which are integrals of the field quantities. The product $\underline{\mathcal{V}} \cdot \mathrm{j} \omega \mathcal{I}$ is a power.

Reversible transducer models for piezoelectric and piezomagnetic two-layer bimorphs are derived next. Table 2 lists the coordinates used in the electrical and magnetic domain. Due to Eq. (61), no distinction is made between a beam of length $l$ and a beam element of length $\Delta x_{1}$.

In accordance with terms used in material science, the indices used in material equations relate direction 3 to the active direction. The convention will be clarified in the context of the analyzed examples.
Table 2. Electrical and magnetic quantities and parameters

\begin{tabular}{lcc}
\hline Quantity/parameter & Electric & Magnetic \\
\hline Across coordinate $\underline{\mathcal{V}}$ & $\underline{u}=\int_{x_{E}} \underline{E}$ & $\underline{V_{\mathrm{m}}}=\int_{x_{H}} \underline{H}$ \\
\hline Time-integrated flow $\underline{\mathcal{I}}$ & $\underline{Q}=\iint_{A} \underline{D}$ & $\underline{\Phi}=\iint_{A} \underline{B}$ \\
\hline Flow coordinate $\mathrm{d} \underline{\mathcal{I}} \mathrm{d} t$ & $\underline{i}$ & $\underline{I}_{\mathrm{m}}$ \\
\hline
\end{tabular}

\subsection{Piezoelectric bimorph}

Using Einstein notation, piezoelectric state equations are written as

$$
\begin{aligned}
& \underline{D}_{n}=\varepsilon_{n m}^{T} \underline{E}_{m}+d_{n j} \underline{T}_{j} \quad n=1 \ldots 3, m=1 \ldots 3, \\
& j=1 \ldots 6 \text {, } \\
& \underline{S}_{i}=d_{m i} \underline{E}_{m}+s_{i j}^{E} \underline{T}_{j} \quad i=1 \ldots 6, m=1 \ldots 3, \\
& j=1 \ldots 6 \text {, }
\end{aligned}
$$

with displacement current $\underline{D}$, electrical field strength $\underline{E}$, piezoelectric charge constant $d$ and permittivity $\varepsilon$.

Two typical electrode arrangements are considered next.

\subsubsection{Transverse piezoelectric coupling}

An important technical configuration is transverse coupling of two piezoelectric layers as shown in Fig. 15. For a piezoelectric longitudinally acting bimorph with small beam width $\left(\underline{E}_{2}, \underline{E}_{1}=0\right.$ and $\left.\underline{T}_{2} \ldots T_{6}=0\right)$, Eqs. (63) and (64) simplify to

$\underline{D}_{3}=d_{31} \underline{T}_{1}+\varepsilon_{33}^{T} \underline{E}_{3}$,

$\underline{S}_{1}=s_{11}^{E} \cdot \underline{T}_{1}+d_{13} \cdot \underline{E}_{3}$,

$\underline{S}_{2}=s_{21}^{E} \cdot \underline{T}_{1}+d_{23} \cdot \underline{E}_{3}$.

The indices match the directional conventions in Fig. 2. The deformation of the small beam in direction 2 is not further considered. In the case of a large beam width this deformation is suppressed $\left(\underline{S}_{2}=0\right)$, thus causing $\underline{T}_{2} \neq 0$, such that

$\underline{S}_{1}=s_{11}^{E} \cdot \underline{T}_{1}+s_{12}^{E} \cdot \underline{T}_{2}+d_{13} \cdot \underline{E}_{3}$,

$\underline{S}_{2}=s_{21}^{E} \cdot \underline{T}_{1}+s_{22}^{E} \cdot \underline{T}_{2}+d_{23} \cdot \underline{E}_{3}=0$,

$\underline{D}_{3}=d_{31} \cdot \underline{T}_{1}+d_{32} \cdot \underline{T}_{2}+\varepsilon_{33}^{T} \cdot \underline{E}_{3}$

or, including $s_{12}^{E}=s_{21}^{E}$ and $d_{23}=d_{32}$, 


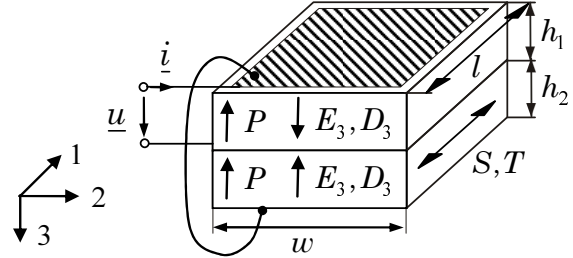

Figure 15. Transversely coupled piezoelectric two-layer beam.

$\underline{S}_{1}=\underbrace{\left(s_{11}^{E}-\frac{\left(s_{12}^{E}\right)^{2}}{s_{22}^{E}}\right)}_{s_{11}^{E *}} \underline{T}_{1}+\underbrace{\left(d_{13}-\frac{d_{23} s_{12}^{E}}{s_{22}^{E}}\right)}_{d_{13}^{*}} \underline{E}_{3}$,

(68a)

$\underline{D}_{3}=\underbrace{\left(d_{31}-\frac{d_{32} s_{21}^{E}}{s_{22}^{E}}\right)}_{d_{31}^{*}} \underline{T}_{1}+\underbrace{\left(\varepsilon_{33}^{T}-\frac{d_{32}^{2}}{s_{22}^{E}}\right)}_{\varepsilon_{33}^{T *}} \underline{E}_{3}$

can be written. Equations (66a) and (68a) differ only in the coefficients. $\underline{T}_{1}, \underline{S}_{1}$ and $\underline{E}_{3}$ are functions of $x_{3}$ while the displacement current $\underline{D}_{3}$ does not depend on coordinate $x_{3}$ because of $\operatorname{div} \mathbf{D}=0$. When these equations are rearranged to $(\underline{T}, \underline{E}=f(\underline{S}, \underline{D}))($ Lenk et al., 2011, pp. 352)

$\underline{T}_{1}=\frac{1}{s_{11}^{E}\left(1-k_{31}^{2}\right)} \cdot \underline{S}_{1}-\frac{1}{d_{31}}\left(\frac{k_{31}^{2}}{1-k_{31}^{2}}\right) \cdot \underline{D}_{3}$,

$\underline{E}_{3}=-\frac{1}{d_{31}}\left(\frac{k_{31}^{2}}{1-k_{31}^{2}}\right) \cdot \underline{S}_{1}+\frac{1}{\varepsilon_{33}^{T}\left(1-k_{31}^{2}\right)} \cdot \underline{D}_{3}$,

with

$k_{31}^{2}=\frac{d_{31}^{2}}{\varepsilon_{33}^{T} s_{11}^{E}}$,

moment $\underline{M}\left(x_{1}\right)$ and force $\underline{F}_{1}\left(x_{1}\right)$ follow from Eqs. (13) and (14) with

$\underline{i}_{1}=\mathrm{j} \omega A \underline{D}_{3,1} \quad$ and $\quad \underline{i}_{2}=\mathrm{j} \omega A \underline{D}_{3,2}$,

where $A=w \cdot l$ is the through-flow area

$$
\begin{aligned}
\underline{M} & =-\frac{1}{\mathrm{j} \omega} \underbrace{\frac{\overline{E I}}{l}}_{1 / n_{\mathrm{R}, 0}} \underline{\Omega}-\underbrace{\frac{E_{1}}{\mathrm{j} \omega 2 l} \alpha_{1}\left(-h_{1}^{2}+2 c h_{1}\right)}_{-1 / \underline{X}_{\mathrm{R} \perp 1}} \underline{i}_{1} \\
& \underbrace{-\frac{E_{2}}{\mathrm{j} \omega 2 l} \alpha_{2}\left(h_{2}^{2}+2 c h_{2}\right)}_{-1 / \underline{X}_{\mathrm{R} \perp 2}} \underline{i}_{2},
\end{aligned}
$$

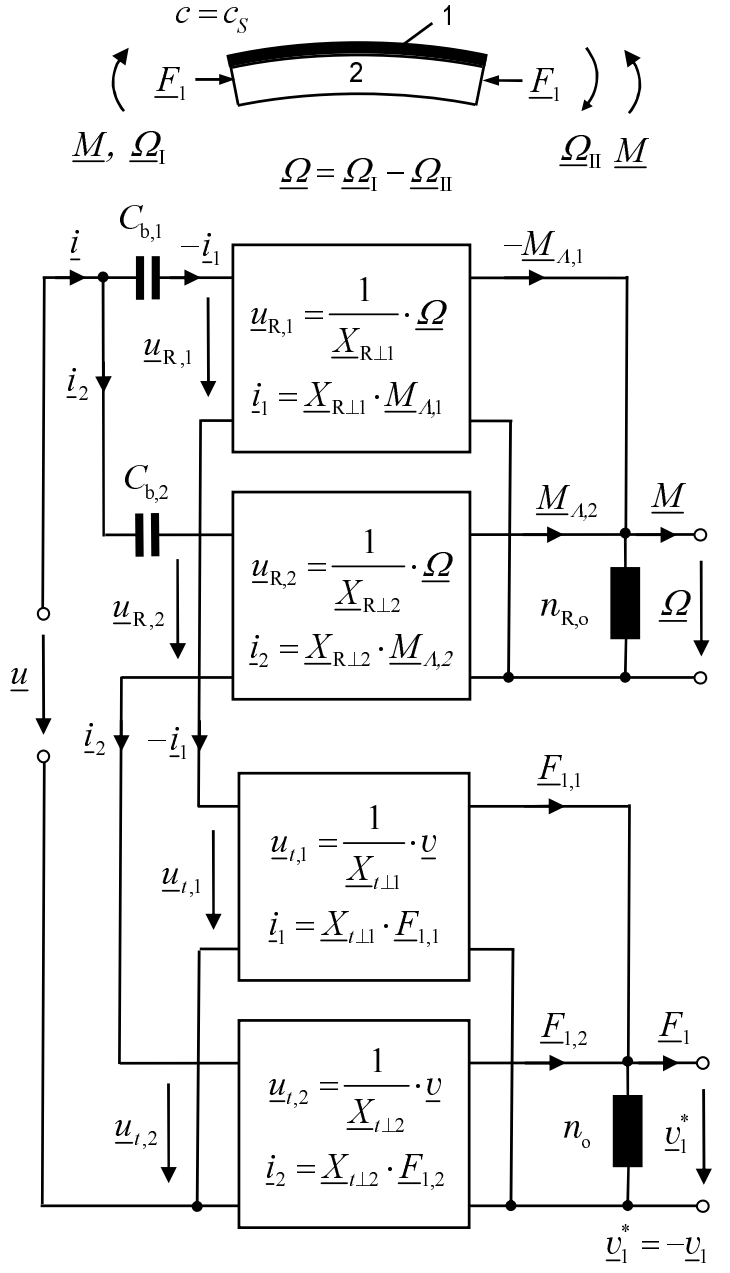

Figure 16. Transversely coupled piezoelectric transducer model given by Eqs. (75), (72) and (73).

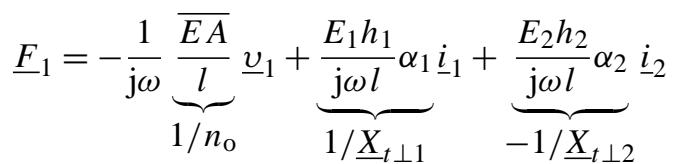

and with the material parameters $\alpha=-d_{31} / \varepsilon_{33}^{T}$ and

$$
E=\frac{1}{s_{11}^{E}\left(1-k_{31}^{2}\right)} \text {. }
$$

Equations (72) and (73) describe a moment node and a force node including the complex translational and rotational transduction coefficients $\underline{X}_{t \perp}$ and $\underline{X}_{\mathrm{R} \perp}$, from which the mechanical domain in Fig. 16 can be deduced. The setup-related directions of $\underline{i}_{1}, \underline{M}_{\Lambda, 1}$ and $\underline{v}_{1}$ were reversed to match the directions of the arrows in Fig. 14.

Integrating $\underline{E}$ over $x_{3}$ in each layer - designated by subscript , 1 and ,2 or $\perp 1$ and $\perp 2$ - and considering Eqs. (44), 
(45), (69b) and (10) gives the voltage

$$
\begin{aligned}
& \underline{u}=-\int_{c-h_{1}}^{c} \underline{E}_{3,1} \mathrm{~d} x_{3}=\int_{c}^{c+h_{2}} \underline{E}_{3,2} \mathrm{~d} x_{3} \\
& =-\int_{c-h_{1}}^{c}\left(E_{1} \alpha_{1} \frac{\mathrm{d} \underline{\xi}_{1}}{\mathrm{~d} x_{1}}+E_{1} \alpha_{1} x_{3} \frac{\mathrm{d} \underline{\varphi}}{\mathrm{d} x_{1}}+\beta_{1}^{S} \underline{D}_{3,1}\right) \mathrm{d} x_{3} \\
& =\int_{c}^{c+h_{2}}\left(E_{2} \alpha_{2} \frac{\mathrm{d} \underline{\xi}_{1}}{\mathrm{~d} x_{1}}+E_{2} \alpha_{2} x_{3} \frac{\mathrm{d} \underline{\varphi}}{\mathrm{d} x_{1}}+\beta_{2}^{S} \underline{D}_{3,2}\right) \mathrm{d} x_{3} \\
& =\underbrace{\frac{E_{1} h_{1}}{\mathrm{j} \omega l} \alpha_{1}}_{1 / \underline{X}_{t \perp 1}} \underline{v}_{1}+\underbrace{\frac{E_{1}}{\mathrm{j} \omega 2 l} \alpha_{1}\left(-h_{1}^{2}+2 c h_{1}\right)}_{-1 / \underline{X}_{\mathrm{R} \perp 1}} \underline{\Omega} \\
& -\frac{1}{\mathrm{j} \omega} \underbrace{\frac{h_{1} \beta_{1}^{S}}{w l}}_{1 / C_{b, 1}} i_{1} \\
& =-\underbrace{\frac{E_{2} h_{2}}{\mathrm{j} \omega l} \alpha_{2}}_{-1 / \underline{X}_{t \perp 2}} \underline{v}_{1} \underbrace{-\frac{E_{2}}{\mathrm{j} \omega 2 l} \alpha_{2}\left(h_{2}^{2}+2 c h_{2}\right)}_{-1 / \underline{X}_{\mathrm{R} \perp 2}} \underline{\Omega} \\
& +\frac{1}{\mathrm{j} \omega} \underbrace{\frac{h_{2} \beta_{2}^{S}}{w l}} \underline{i}_{2} \text {, } \\
& 1 / C_{b, 2}
\end{aligned}
$$

with

$\beta^{S}=1 /\left(\varepsilon_{33}^{T}\left(1-k_{31}^{2}\right)\right)$.

The resulting voltage meshes in Fig. 16 include the electrical capacitances $C_{b, 1}$ and $C_{b, 2}$ of the two layers.

The capacitance $C_{b}=C_{b, 1}+C_{b, 2}$ can be measured in the case of the blocked beam $(\underline{v}=\underline{\Omega}=0)$.

Compliance $n_{\mathrm{o}}$ and rotational compliance $n_{\mathrm{R}, \mathrm{o}}$ are labeled with the subscript "o" for "open" since they could be measured in the case of no current $\left(\underline{i}_{1}=\underline{i}_{2}=0\right)$. If the two layers are nonsymmetric, then this boundary condition cannot be realized. The open electrical circuit connects $C_{b, 1}$ and $C_{b, 2}$ in series, which allows a current flow. This connection provides a link between rotation and translation. The transfer function $\underline{v}_{1}^{*} /\left.\underline{\Omega}_{0}\right|_{\underline{i}=0}$ for this case can be determined analytically for the circuit in Fig. 16, which is simplified for this purpose. The first step is to explore the effect of an assumed rotational velocity source $\underline{\Omega}_{0}$ in the electrical domain. The effect can be found by a source transformation as demonstrated in Fig. 17. The electrorotational transducers can be ignored afterwards since they do not influence the ideal sources and current $\underline{i}_{1}$ which flows through the electrotranslational transducers. The result of this reduction step is depicted in Fig. 18a. The circuit can be simplified further, when the combined source

$\underline{u}_{\Omega}=\left(\frac{1}{\underline{X}_{\mathrm{R} \perp 1}}-\frac{1}{\underline{X}_{\mathrm{R} \perp 2}}\right) \cdot \underline{\Omega}_{0}$

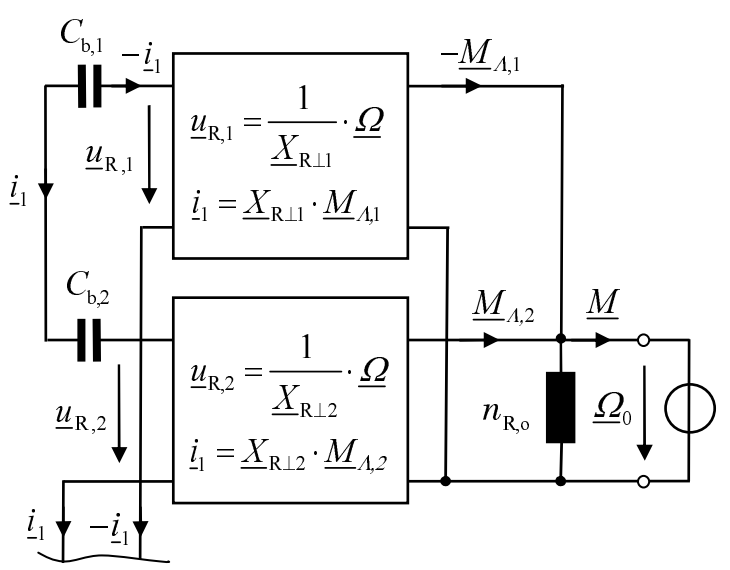

Transformation $\square$

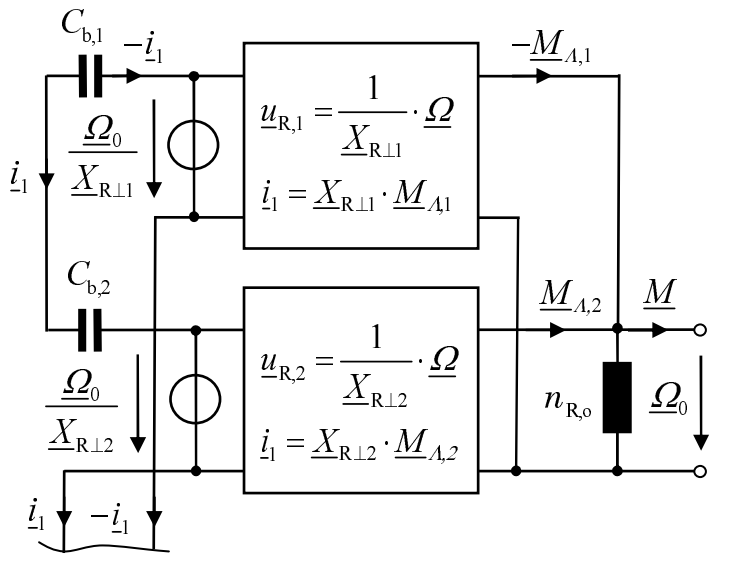

Figure 17. Transformation of a rotational velocity source.

and total capacitance

$C_{\mathrm{ser}}=\frac{C_{b, 1} \cdot C_{b, 2}}{C_{b, 1}+C_{b, 2}}$

are transformed into the translational mechanical domain. The complex transduction coefficient causes a transition to a compliant behavior or inductance symbol. Then the two transducers are solely connected to each other. They can be combined into a lever with the ratio or mechanical advantage

$t=\frac{\underline{X}_{t \perp 1}}{\underline{X}_{t \perp 2}}$

as depicted in Fig. 18b. The sought transfer function:

$\left.\underline{\underline{\Omega_{1}}} \underline{\underline{\Omega}}_{0}\right|_{\underline{i}=0}=\frac{\underline{X}_{t \perp 1} \cdot\left(\frac{1}{\underline{X}_{\mathrm{R}} \perp 1}-\frac{1}{\underline{X}_{\mathrm{R}} \perp 2}\right)}{t-1+\frac{n_{C}}{n_{\mathrm{o}} \cdot(t-1)}}$

with

$n_{C}=-\frac{\underline{X}_{t \perp 1}^{2}}{\omega^{2} C_{\mathrm{ser}}}$, 


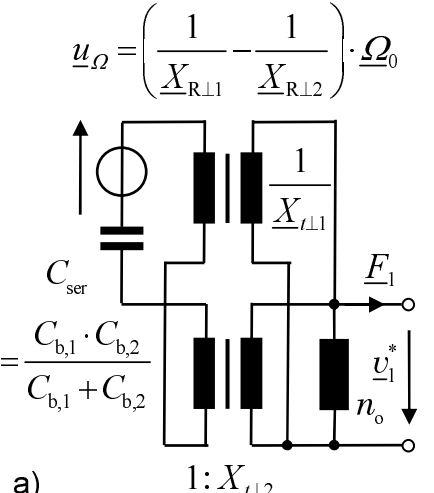

a)

$1: \underline{X}_{t \perp 2}$

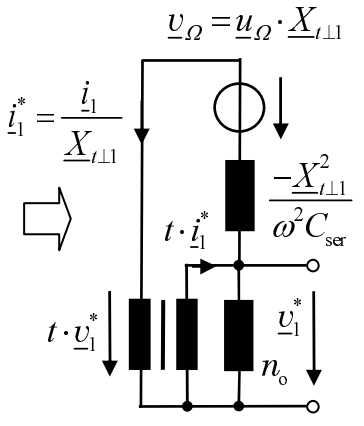

b) $\quad t=\underline{X}_{t \perp 1}: \underline{X}_{t \perp 2}$

Figure 18. Length variation of a transversally coupled piezoelectric bimorph caused by a rotational velocity.

is found by applying Kirchhoff's laws:

O: $-\underline{v}_{1}^{*}+\mathrm{j} \omega n_{C} \cdot \frac{\underline{i}_{1}}{\underline{X}_{t \perp 1}}-\underline{v}_{\Omega}+t \cdot \underline{v}_{1}^{*}=0$,

$\circledast: t \cdot \underline{i}_{1}^{*}-\underline{i}_{1}^{*}-\frac{\underline{v}_{1}^{*}}{\mathrm{j} \omega n_{0}}=0$,

with

$\underline{i}_{1}^{*}=\frac{\underline{i}_{1}}{\underline{X}_{t \perp 1}}$.

No dependency occurs when $\underline{X}_{\mathrm{R} \perp 1}=\underline{X}_{\mathrm{R} \perp 2}$.

\subsubsection{Symmetric laminate}

A special case of transverse coupling is the laminate of two equal piezoelectric layers as shown in Fig. 15 where $\underline{i}_{1}=$ $-\underline{i}_{2}=\underline{i} / 2$ holds. Such a symmetric laminate is characterized by $\underline{X}_{\mathrm{R} \perp 1}=\underline{X}_{\mathrm{R} \perp 2}$ and $\underline{X}_{t \perp 1}=\underline{X}_{t \perp 2}$, i.e., $t=1$. Simplified network parameters - as derived by Lenk and Irrgang (1977) - result from Eqs. (75), (72) and (73):

$\underline{M}=-\frac{1}{\mathrm{j} \omega n_{\mathrm{R}, \mathrm{o}}} \cdot \underline{\Omega}+\frac{1}{\underline{X}_{\mathrm{R} \perp}} \cdot \underline{i}$,

$\underline{F}_{1}=-\frac{1}{\mathrm{j} \omega n} \cdot \underline{v}$,

$$
\begin{aligned}
\underline{u} & =-\frac{1}{\underline{X}_{t \perp 1}} \cdot \underline{v}+\frac{1}{\underline{X}_{\mathrm{R} \perp 1}} \cdot \underline{\Omega}+\frac{1}{\mathrm{j} \omega C_{b, 1}} \cdot \frac{\underline{i}}{2} \\
& =-\frac{1}{\underline{X}_{t \perp 2}} \cdot \underline{v}+\frac{1}{\underline{X}_{\mathrm{R} \perp 2}} \cdot \underline{\Omega}+\frac{1}{\mathrm{j} \omega C_{b, 2}} \cdot \frac{i}{2},
\end{aligned}
$$

with

$n_{\mathrm{R}, \mathrm{o}}=\frac{E I}{l}$,

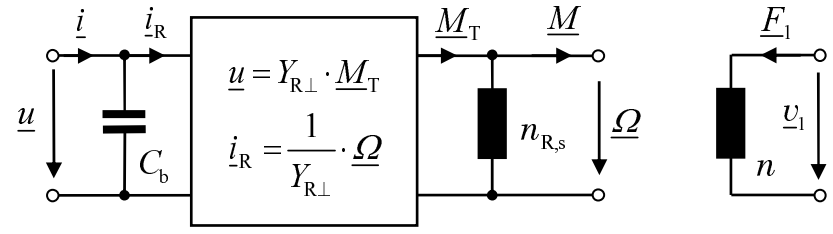

Figure 19. Model of a transversely coupled symmetric piezoelectric two-layer beam.

$$
I=\frac{w\left(2 h_{1}\right)^{3}}{12}
$$

$\frac{1}{\underline{X}_{\mathrm{R} \perp}}=\frac{3}{4} \frac{n_{\mathrm{R}, \mathrm{o}}}{w h_{1}} \frac{\alpha}{\mathrm{j} \omega}$,

$n=\frac{E A}{l}$

and

$A=2 w h_{1}$.

The neutral layer is located at the material interface, i.e., $c_{A}=c_{S}$. Therefore, no translational electromechanical effect occurs $\left(\underline{F}_{1} \neq f(\underline{i})\right)$. In the sensing case, opposite voltages are caused by $\underline{v}_{1}$ which cancel each other out $\left(\underline{u} \neq f\left(\underline{v}_{1}\right)\right)$.

Following Lenk and Irrgang (1977), a circuit description with real transduction coefficients

$\frac{1}{Y_{\mathrm{R} \perp}}=\frac{\mathrm{j} \omega C_{b}}{X_{\mathrm{R} \perp}}=\frac{d_{31}}{s_{11}^{E}} w h_{1}$

is obtained by rearranging SOE (85) to

$$
\begin{aligned}
\underline{M} & =-\frac{1}{\mathrm{j} \omega n_{\mathrm{R}, \mathrm{s}}} \cdot \underline{\Omega}+\frac{1}{Y_{\mathrm{R} \perp}} \cdot \underline{u} \\
\underline{i} & =\frac{1}{Y_{\mathrm{R} \perp}} \cdot \underline{\Omega}+\mathrm{j} \omega C_{\mathrm{b}} \cdot \underline{u},
\end{aligned}
$$

where $n_{\mathrm{R}, \mathrm{s}}$ is the short-circuit compliance (subscript "s")

$n_{\mathrm{R}, \mathrm{s}}=\frac{l}{E I} \frac{1}{\left(1-\frac{3}{4} k_{31}^{2}\right)}$,

and

$C_{b}=C_{b, 1}+C_{b, 2}=2 \varepsilon_{33}^{T}\left(1-k_{31}^{2}\right) \frac{l w}{h_{1}}$

is the total blocked capacitance. SOE (92) can be interpreted as the circuit in Fig. 19 (Lenk et al., 2011). 


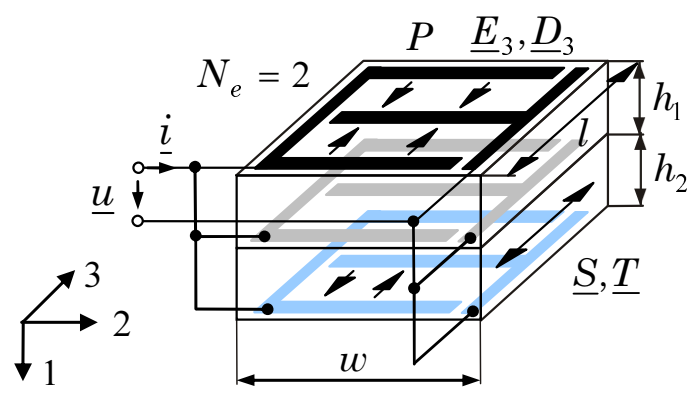

Figure 20. Longitudinally coupled piezoelectric two-layer beam.

\subsubsection{Longitudinal piezoelectric coupling}

Longitudinal piezoelectric coupling is achieved with $\left(N_{\mathrm{e}}+1\right)$ interdigital electrodes on the piezoelectric layers creating $N_{\mathrm{e}}$ separate electric field regions as sketched in Fig. 20. The layers are polarized longitudinally and match the comb structure but with opposite direction in layers 1 and 2 . In the following, thin layers are assumed which accommodate an approximately uniform electric field in direction 1 . The material directions 1 and 3 are now exchanged compared to the coordinate system in Fig. 2.

Longitudinal piezoelectric coupling is described by

$\underline{T}_{3}=\frac{1}{s_{33}^{E *}} \cdot \underline{S}_{3}-\frac{d_{33}^{*}}{s_{33}^{E *}} \cdot \underline{E}_{3}$,

$\underline{D}_{3}=d_{33}^{*} \cdot \underline{T}_{3}+\varepsilon_{33}^{T *} \cdot \underline{E}_{3}$,

matching the abbreviations $1 / E=s_{33}^{E *}=s_{33}^{E}-\left(s_{23}^{E}\right)^{2} / s_{22}^{E}$, $\beta^{T}=\varepsilon_{33}^{T *}=\varepsilon_{33}^{T}-d_{23}^{2} / s_{22}^{E} \quad$ and $\quad \alpha_{1}=-\alpha_{2}=d_{33}^{*}=$ $d_{33}-s_{32}^{E} d_{23} / s_{22}^{E}$, as well as $\underline{\Lambda}=\underline{E}_{3}$.

Calculation of moment $M$ and longitudinal force $F_{3}$ with Eqs. (13) and (14) and $\underline{u}=\underline{E}_{3} \cdot l / N_{\mathrm{e}}$ give the rotational electromechanical transduction coefficient $Y_{\mathrm{R}}$ for the actuation Eq. (21)

$\underline{M}_{\lambda}=\underbrace{-\frac{w}{2} \frac{E_{1} E_{2} h_{1} h_{2}\left(h_{1}+h_{2}\right)}{\left(E_{1} h_{1}+E_{2} h_{2}\right)} \frac{\left(\alpha_{1}-\alpha_{2}\right)}{l / N_{\mathrm{e}}}}_{1 / Y_{\mathrm{R}}} \underline{u}$,

and the translational electromechanical transduction coefficient in the combination of Eq. (42b) and Eqs. (18) and (19):

$$
\begin{aligned}
\frac{1}{Y_{t}}=\left.\frac{\underline{F}_{1}}{\underline{u}}\right|_{\frac{\mathrm{d} \xi_{3}}{\mathrm{~d} x_{3}}=0} & =\frac{\overline{E A} \cdot \bar{\alpha}}{l / N_{\mathrm{e}}} \\
& =\frac{w}{l / N_{\mathrm{e}}}\left(\alpha_{1} E_{1} h_{1}+\alpha_{2} E_{2} h_{2}\right) .
\end{aligned}
$$

As in Sect. 10.1.2 this coefficient is 0 when the two piezoelectric layers are equal and $c_{A}=c_{S}$, too.

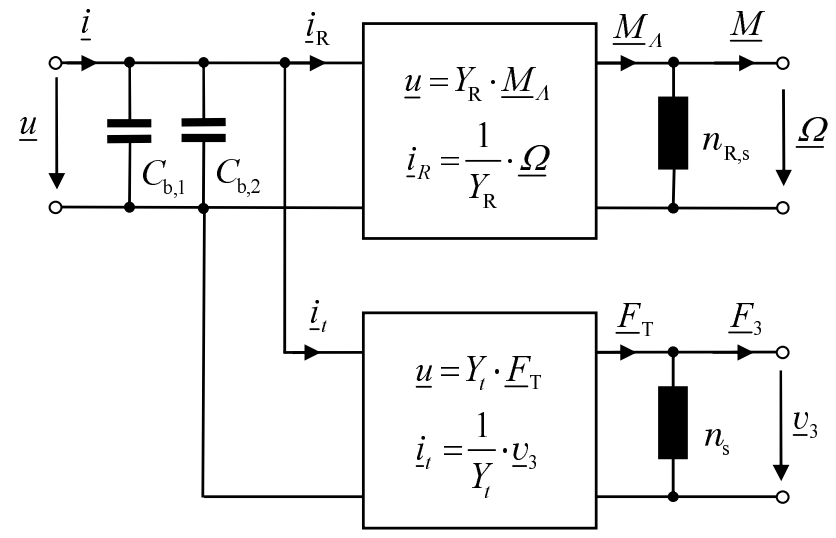

Figure 21. Model of a longitudinally coupled piezoelectric beam.

The time-integrated flow coordinate charge $\underline{Q}$ is gained from the density quantity $\underline{D}_{3}$ by

$$
\begin{aligned}
& \underline{Q}=n \cdot w \int_{c-h_{1}}^{c+h_{2}}\left(\alpha E \frac{\mathrm{d} \underline{\xi}_{3}}{\mathrm{~d} x_{3}}+\alpha E x_{1} \frac{\mathrm{d} \underline{\varphi}}{\mathrm{d} x_{3}}+\left(\beta-\alpha^{2} E\right) \underline{\Lambda}\right) \mathrm{d} x_{1} \\
& =w\left\{\left[\frac{1}{2}\left(\alpha_{2} E_{2} h_{2}^{2}-\alpha_{1} E_{1} h_{1}^{2}\right)+\left(\alpha_{1} E_{1} h_{1}+\alpha_{2} E_{2} h_{2}\right) c\right] \frac{\mathrm{d} \underline{\varphi}}{\mathrm{d} x_{3}}\right. \\
& +\left(\alpha_{1} E_{1} h_{1}+\alpha_{2} E_{2} h_{2}\right) \frac{\mathrm{d} \underline{\xi}_{1}}{\mathrm{~d} x_{3}} \\
& \left.+\left[\left(\beta_{1}-\alpha_{1}^{2} E_{1}\right) h_{1}+\left(\beta_{2}-\alpha_{2}^{2} E_{2}\right) h_{2}\right] \underline{\Lambda}\right\}
\end{aligned}
$$

in the case of a uniform electrical field in direction 2. When curvature, strain along $x_{3}$ and the $\Lambda$ field coordinate are all constant, then the flow coordinate - current $-\underline{i}=\mathrm{j} \omega \underline{Q}$

$$
\begin{aligned}
& \underline{i}=\underbrace{-\frac{w}{2} \frac{E_{1} E_{2} h_{1} h_{2}\left(h_{1}+h_{2}\right)}{\left(E_{1} h_{1}+E_{2} h_{2}\right)} \frac{\left(\alpha_{1}-\alpha_{2}\right)}{l / N_{\mathrm{e}}}}_{1 / Y_{R}} \underline{\Omega} \\
& +\underbrace{\left(\alpha_{1} E_{1} h_{1}+\alpha_{2} E_{2} h_{2}\right) \frac{w}{l / N_{\mathrm{e}}}} \underline{v}_{3} \\
& 1 / Y_{t} \\
& +\mathrm{j} \omega[\underbrace{\left(\beta_{1}^{T}-\alpha_{1}^{2} E_{1}\right) \frac{w h_{1}}{l / N_{\mathrm{e}}}}_{\mathcal{C}_{\mathrm{b}, 1}}+\underbrace{\left(\beta_{2}^{T}-\alpha_{2}^{2} E_{2}\right) \frac{w h_{2}}{l / N_{\mathrm{e}}}}_{\mathcal{C}_{\mathrm{b}, 2}}] \underline{u}
\end{aligned}
$$

includes a bending-induced, a $x_{3}$-translationally induced and a voltage-induced component with the transduction coefficients $Y_{\mathrm{R}}$ and $Y_{t}$ and the layer capacitancies $C_{\mathrm{b}, 1}$ and $C_{\mathrm{b}, 2}$.

Together with Eqs. (42f) and (56), a circuit interpretation of Eqs. (99) and (96) leads to the reversible transducer model in Fig. 21. Short-circuiting the electrical port decouples translational and rotational domain. Then the compliance $n_{\mathrm{s}}$ and rotational compliance $n_{\mathrm{R}, \mathrm{s}}$ can be measured. 


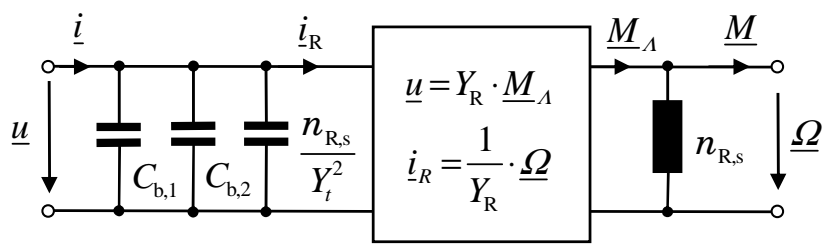

Figure 22. Model of a longitudinally coupled piezoelectric beam with transformed translational compliance.

When only one mechanical domain is of interest, the compliances of the other mechanical domain can be transformed into the electrical domain. As depicted in Fig. 22, the translational compliance, for example, is recognized as an additional capacitance for $\underline{F}_{1}=0$.

\subsection{Piezomagnetic bimorph}

In the case of a piezomagnetic bimorph - analog to piezoelectric materials - the linear magnetomechanical coupling matrix for the field quantities stress $\underline{T}$, strain $\underline{S}$, magnetic flux density $\underline{B}\left(=\underline{\mathcal{K}}_{i}\right)$ and magnetic field strength $\underline{H}(=\underline{\Lambda})$ is given by

$$
\begin{array}{rl}
\underline{B}_{n}=\mu_{n m}^{T} \underline{H}_{m}+d_{n j} \underline{T}_{j} & n=1 \ldots 3, m=1 \ldots 3, \\
& j=1 \ldots 6 \\
\underline{S}_{i}=d_{m i} \underline{H}_{m}+s_{i j}^{H} \underline{T}_{j} & i=1 \ldots 6, m=1 \ldots 3, \\
& j=1 \ldots 6
\end{array}
$$

where $d(=\alpha)$ is the piezomagnetic constant and $\mu(=\beta)$ the permeability.

Technically important electromagnetic transducers are solenoid coils and planar coils.

\subsubsection{Longitudinal coupling with an ideal solenoid}

Longitudinal coupling is achieved when a solenoid coil with $N$ turns around a considered unimorph - with one piezomagnetic layer - serves as an electromagnetic transducer as shown in Fig. 1d. An ideal solenoid coil concentrates a uniform $H$ field in the unimorph and covers the unimorph without an air gap. Demagnetization effects are not considered.

For longitudinal coupling, the magnetomechanical coupling matrix of SOE (100) simplifies for a small beam width to

$$
\begin{aligned}
& \underline{S}_{3}=s_{33}^{H} \cdot \underline{T}_{3}+d_{33} \cdot \underline{H}_{3} \\
& \underline{B}_{3}=d_{33} \cdot \underline{T}_{3}+\mu_{33}^{T} \cdot \underline{H}_{3}
\end{aligned}
$$

and in the case of a large beam width to

$$
\begin{aligned}
& \underline{S}_{3}=s_{33}^{H} \cdot \underline{T}_{3}+s_{31}^{H} \cdot \underline{T}_{1}+d_{33} \cdot \underline{H}_{3} \\
& \underline{S}_{1}=s_{13}^{H} \cdot \underline{T}_{3}+s_{11}^{H} \cdot \underline{T}_{1}+d_{31} \cdot \underline{H}_{3}=0 \\
& \underline{B}_{3}=d_{33} \cdot \underline{T}_{3}+d_{31} \cdot \underline{T}_{1}+\mu_{33}^{T} \cdot \underline{H}_{3}
\end{aligned}
$$

Magnetic flux

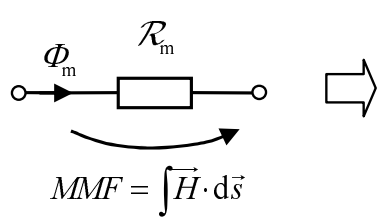

Magnetomotive Force

$$
M M F=\mathcal{R}_{\mathrm{m}} \cdot \Phi_{\mathrm{m}} \quad \underline{V}_{\mathrm{m}}=\frac{\mathcal{R}_{\mathrm{m}}}{\mathrm{j} \omega} \cdot \underline{I}_{\mathrm{m}}
$$

Magnetic flux rate

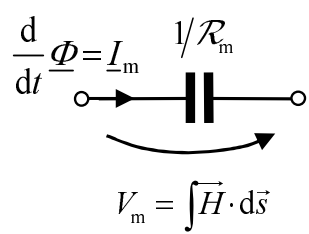

Magnetic Voltage

Figure 23. Magnetic coordinates on a reluctance.

or

$$
\begin{gathered}
\underline{S}_{3}=\underbrace{\left(s_{33}^{H}-\frac{\left(s_{31}^{H}\right)^{2}}{s_{11}^{H}}\right)}_{s_{31}^{H *}} \underline{T}_{3}+\underbrace{\left(d_{33}-\frac{d_{31} s_{31}^{H}}{s_{11}^{H}}\right)}_{d_{33}^{*}} \underline{H}_{3} \\
\underline{B}_{3}=\underbrace{\left(d_{33}-\frac{d_{31} s_{13}^{H}}{s_{11}^{H}}\right)}_{d_{33}^{*}} \underline{T}_{3}+\underbrace{\left(\mu_{33}^{T}-\frac{d_{31}^{2}}{s_{11}^{H}}\right)}_{\mu_{33}^{T *}} \underline{H}_{3} .
\end{gathered}
$$

In the magnetic domain, magnetic voltage $\underline{V}_{\mathrm{m}}$ and the time derivative of the magnetic flux $\mathrm{d} \Phi / \mathrm{d} t$, or magnetic flux rate $\underline{I}_{\mathrm{m}}$, are chosen as coordinates as listed in Table 2. An ideal solenoid coil then acts as electromechanical transducer with coefficient $N$, which is coupled with the piezomagnetic beam (Marschner et al., 2010). With magnetic voltage and flux rate, a magnetic reluctance $\mathcal{R}_{\mathrm{m}}$ is described by the capacitor symbol due to the analogy with the capacitance $\underline{u}=1 /(\mathrm{j} \omega C) \cdot \underline{i}$ as explained in Fig. 23.

With the abbreviations $E=1 / s_{33}^{E}, \alpha=d_{33}$ and $\beta^{T}=\mu_{33}^{T}$, the transduction coefficients are obtained by Eqs. (96) and (97) when $N_{\mathrm{e}}$ is set to 1 , and in addition to the material coefficients, $\underline{u}$ is substituted by $\underline{V}_{\mathrm{m}}$ and $\underline{\Lambda}$ by $\underline{H}_{3}$. Also replacing $Q$ by the magnetic flux $\underline{\Phi}$, the magnetic flux rate $\underline{I}_{\mathrm{m}}$ instead of $i$ follows with Eq. (99), where the reluctances of the layers $\mathcal{R}_{\mathrm{m}, \mathrm{b}, 1}$ and $\mathcal{R}_{\mathrm{m}, \mathrm{b}, 2}$ can be measured when the bimorph is mechanically blocked. Figure 24 shows the resulting electromechanical circuit representation. Compliance $n$ and rotational compliance $n_{\mathrm{R}}$ are separated in case of a suppressed magnetic voltage variation $\left(\underline{V}_{\mathrm{m}}=0\right)$ which is achieved by an open electrical circuit $(\underline{i}=0)$.

The model is valid for small variations of the magnetic and mechanical system quantities. In order to model nonlinear sensing and actuating behaviors of piezomagnetic materials, finite-element-based models comprise magnetic and elastic boundary value problems (BVPs) that are bidirectionally coupled through stress and field-dependent coupling variables - magnetostriction and magnetization (Mudivarthi et al., 2008). 


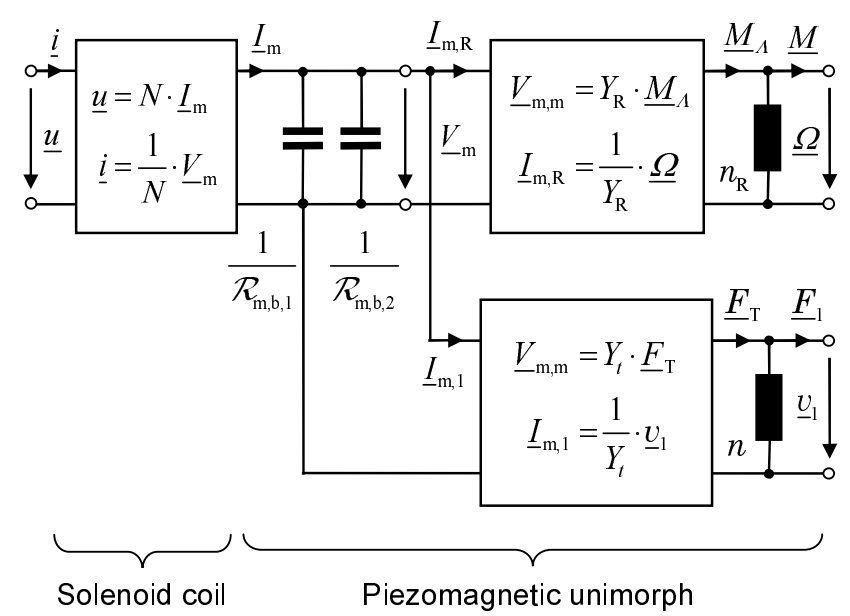

Figure 24. Circuit model for uniform longitudinal electromagnetic coupling achieved by applying an ideal solenoid coil.

\subsubsection{Transverse coupling}

The linearized constitutive equations for transverse coupling of mechanical and magnetic field quantities for a small beam can be formulated as

$$
\begin{gathered}
\underline{S}_{1}=d_{13} \cdot \underline{H}_{3}+s_{11}^{H} \cdot \underline{T}_{1} \\
\underline{B}_{3}=\mu_{33}^{T} \cdot \underline{H}_{3}+d_{31} \cdot \underline{T}_{1}
\end{gathered}
$$

or

$\underline{T}_{1}=-\frac{d_{13}}{s_{11}^{H}} \cdot \underline{H}_{3}+\frac{1}{s_{11}^{H}} \cdot \underline{S}_{1}$

$\underline{B}_{3}=\left(\mu_{33}^{T}-\frac{d_{13}^{2}}{s_{11}^{H}}\right) \cdot \underline{H}_{3}+\frac{d_{13}}{s_{11}^{H}} \cdot \underline{S}_{1}$.

Similar considerations for a large beam width hold as discussed for longitudinal coupling.

A technical solution to realizing transverse coupling are planar coils. In a part of the coil the mechanical and magnetic quantities are directed perpendicular to each other as visualized by Fig. 25. When a uniform magnetic field strength in the piezomagnetic layer is assumed, then network parameters similar to longitudinal coupling are found except with the length $l$ and width $w$ of the beam being exchanged as listed in Table 4.

\subsubsection{Example: transverse coupling with planar coil}

Next, the technically interesting case of a thin piezomagnetic layer on a nonmagnetic carrier in combination with a planar set of inductors will be discussed. The planar set of inductors, as shown in Fig. 26, serves as electromagnetic transducer. It can be viewed as a section of a rectangular planar coil. In this specific example a thin isolation layer of $0.2 \mathrm{~mm}$ thickness is located between the conductor layer and the magnetic layer.

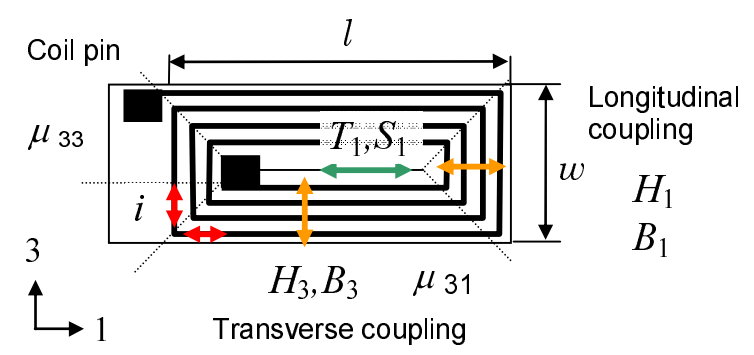

Figure 25. Rectangular planar coil coupling.

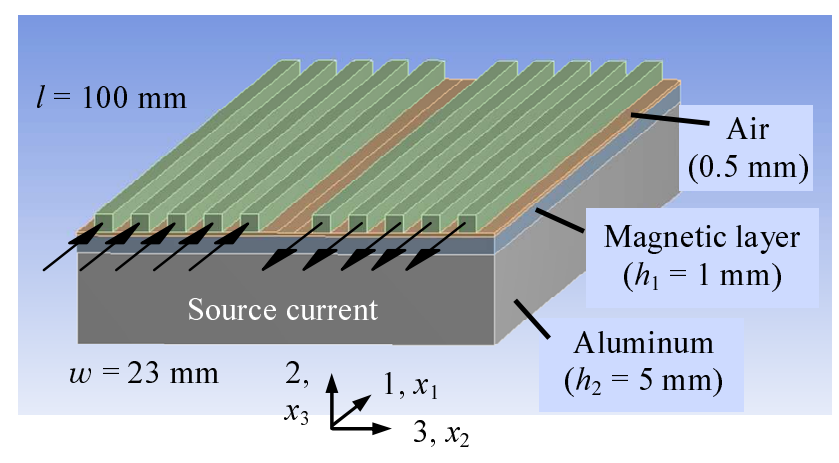

Figure 26. Section of a planar coil on top of a thin piezomagnetic layer.

\section{(a) Coil inductance}

The placement of the ferromagnetic material beside the planar turns has much less influence on the coil inductance or electromagnetic properties in general than a ferromagnetic core inside a solenoid coil. While the inductance of a solenoid coil with isotropic core material depends linearly on the permeability, the inductance of a planar coil $L_{\infty}$ is doubled at the maximum by an isotropic permeable layer of infinite thickness underneath the turns compared to the air coil inductance $L_{0}$. For an infinite layer thickness, Roshen and Turcotte (1988) showed that

$L_{\infty}=\frac{2 \mu_{r}}{\mu_{r}+1} L_{0}$.

It should be noted, that already a relative permeability $\mu_{r}=$ 20 increases $L_{0}$ by about a factor of 1.9. The network model of the coil arrangement, which is derived next, relates the magnetic reluctances to the inductance. This will give an explanation for the inductance peculiarity.

\section{(b) Combined Simulation}

The electromagnetic system is studied for the setup in Fig. 26 using finite-element (FE) simulations to determine the structures and parameters of the magnetic network. The method of deriving network parameters from FE simulations is called Combined Simulation (Starke, 2009), (Starke et al., 2011). The network elements of the circuit in Fig. 29 can be 


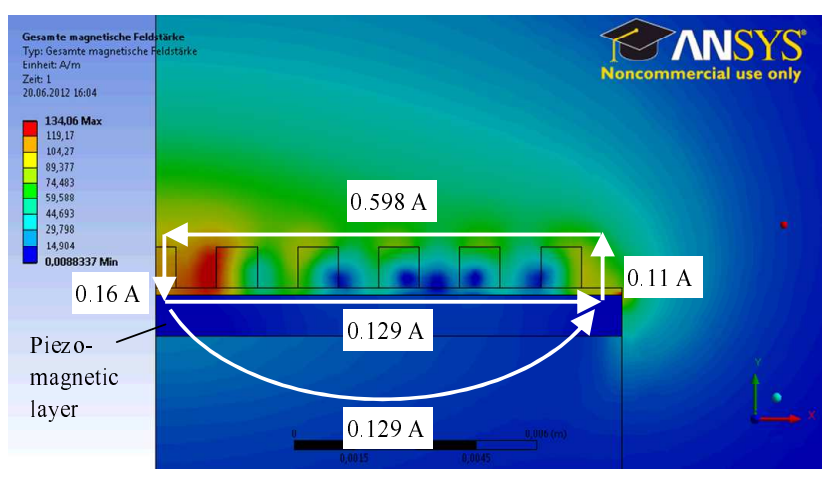

Figure 27. Absolute value of the magnetic field strength $H$ in the right half and magnetic voltages across each path $\mu_{r}=20$.

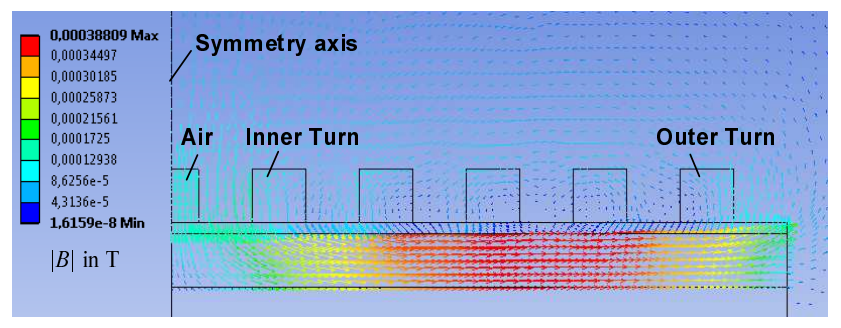

Figure 28. Concentration of the magnetic flux in the magnetic layer near the turns.

determined individually and separately by applying particular boundary conditions (e.g., short or open circuits, excitation quantities). The same conditions are used as boundary conditions for the FE simulations to determine the element values from the complex geometries and field distributions.

A magnetostatic FE analysis, not considering any eddy current losses, reveals the actuation situation when only the low-frequency properties of the unimorph are of interest. The generated magnetic field strength is depicted in Fig. 27. The magnetic flux is concentrated near the turns with a slight peak near the middle of the turn area while the flux in $x_{3}$ direction is nearly uniform (Fig. 28).

\section{(c) Equivalent circuit}

When each partial magnetic voltage along a path around the conductors is related to a reluctance, then a magnetic voltage divider is found where the magnetic voltages are proportional to the reluctances. Due to the ideally equal magnetic voltage in a thin piezomagnetic layer and in the nonmagnetic substrate and air below, as depicted in Fig. 27, the related reluctances $\mathcal{R}_{\mathrm{m}, \mathrm{m}}$ and $\mathcal{R}_{\mathrm{m}, \mathrm{A}, \mathrm{b}}$ are connected in parallel. The equivalent circuit of Fig. 24 can thus be extended by the magnetic voltage divider including reluctance $\mathcal{R}_{\mathrm{m}, \mathrm{A}, \mathrm{a}}$ of the air above and beside the conductors, as shown in Fig. 29. Contrary to the ideal solenoid coupling, the magnetic voltage $\underline{V}_{\mathrm{m}}$ across the magnetic layer cannot be suppressed by setting $\underline{i}=0$. Therefore, $n_{\mathrm{R}}$ and $n$ cannot be measured separately,

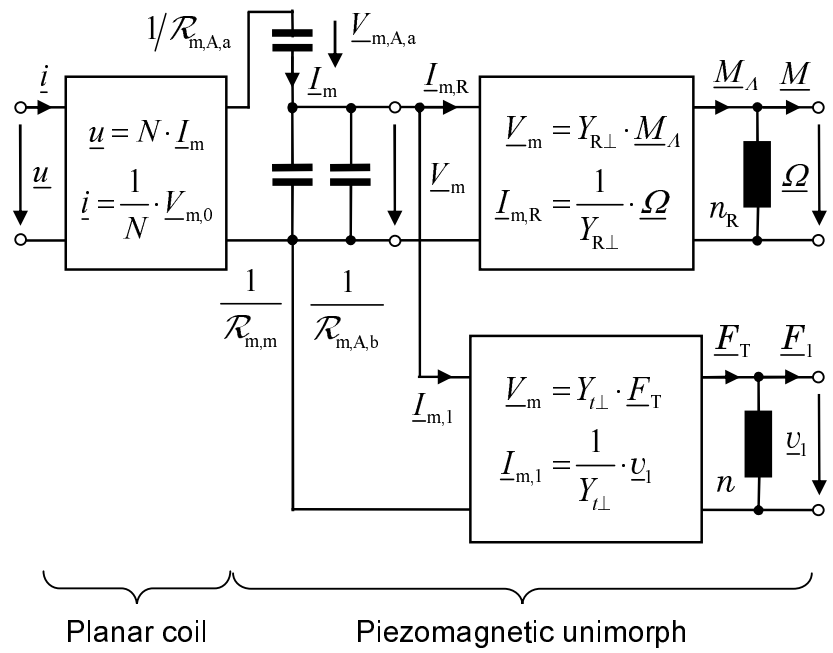

Figure 29. Circuit model for uniform transversal electromagnetic coupling applying an ideal planar conductor setup on a single piezomagnetic layer as shown in Fig. 26.

Table 3. Material parameters of the magnetic layer.

\begin{tabular}{ll}
\hline$d_{33}=10 \cdot 10^{-9} \mathrm{~m} \mathrm{~A}^{-1}$ & $d_{31}=-5 \cdot 10^{-9} \mathrm{~m} \mathrm{~A}^{-1}$ \\
\hline$s_{33}^{H}=3.8 \cdot 10^{-11} \mathrm{~m}^{2} \mathrm{~N}^{-1}$ & $s_{11}^{H}=4.4 \cdot 10^{-11} \mathrm{~m}^{2} \mathrm{~N}^{-1}$ \\
\hline$s_{12}^{H}=-1.1 \cdot 10^{-11} \mathrm{~m}^{2} \mathrm{~N}^{-1}$ & $s_{13}^{H}=-1.65 \cdot 10^{-11} \mathrm{~m}^{2} \mathrm{~N}^{-1}$ \\
\hline$s_{44}^{H}=24 \cdot 10^{-11} \mathrm{~m}^{2} \mathrm{~N}^{-1}$ & $s_{66}^{H}=11 \cdot 10^{-11} \mathrm{~m}^{2} \mathrm{~N}^{-1}$ \\
\hline
\end{tabular}

and the rotational and translational domain cannot be decomposed this way but only when the magnetic reluctances are taken into account (Marschner et al., 2014).

\section{(d) Reluctances}

The reluctances can be determined on the basis of the total inductance of the setup as described by Marschner et al. (2010). The reluctances are separated by a disabled magnetomechanical interaction, which is achieved either by $\underline{\Omega}=0$ and $\underline{v}=0$ or $\underline{I}_{\mathrm{m}, \mathrm{R}}=0$ and $\underline{I}_{\mathrm{m}, 1}=0$. With a pure magnetic FE analysis these ideal boundary conditions are matched. Once the inductance $L$ of the arrangement is determined by FE simulations, the total reluctance can be related to the individual reluctances by applying the magnetic voltage divider rule as shown in Fig. 30. The total reluctance $\mathcal{R}_{\mathrm{m}}=\mathcal{R}_{\mathrm{m}, 1}+\mathcal{R}_{\mathrm{m}, 2}$ and total inductance $L$ are related to each other by

$\frac{N^{2}}{\mathcal{R}_{\mathrm{m}}}=L$

A total inductance of $1.1 \mu \mathrm{H}$ of the electromagnetic system is calculated with FE analysis from the static magnetic field energy. The magnetic voltages across $\mathcal{R}_{\mathrm{m}, 1}$ and $\mathcal{R}_{\mathrm{m}, 2}$ reach $0.871 \mathrm{~A}$ and $0.129 \mathrm{~A}$, as noted in Fig. 27. Back 


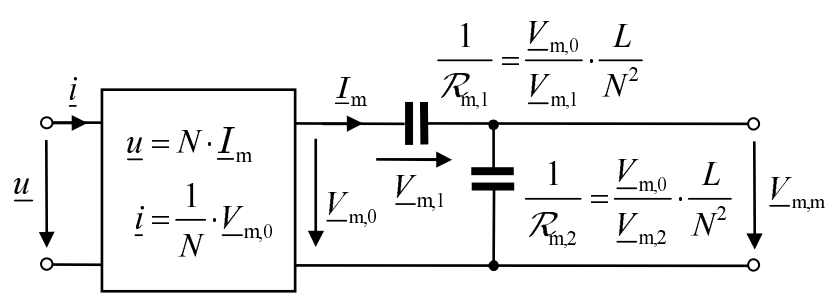

Figure 30. Reluctance calculation based on the inductance. The magnetomechanical system is switched off by blocking the unimorph.

transformation gives $\mathcal{R}_{\mathrm{m}, 1}=20 \mathrm{MA} \mathrm{Wb}^{-1}$ and $\mathcal{R}_{\mathrm{m}, 2}=$ $\mathcal{R}_{\mathrm{m}, \mathrm{A}, \mathrm{b}} \| \mathcal{R}_{\mathrm{m}, \mathrm{m}}=3 \mathrm{MA} \mathrm{Wb}^{-1}$. Assuming a homogeneous field in the magnetic layer, its dimension-based reluctance is

$\mathcal{R}_{\mathrm{m}, \mathrm{m}}=\frac{w_{a}}{\mu_{0} \mu_{r} h_{1} l}=4 \mathrm{MA} \mathrm{Wb}^{-1}$

where $w_{a}=N \cdot\left(w_{t}+d_{w}\right)$ is the width of the field concentration underneath the $N$ turns with width $w_{t}$ and distance $d_{w}$ from each other. It follows, that

$\mathcal{R}_{\mathrm{m}, \mathrm{A}, \mathrm{b}}=\frac{\mathcal{R}_{\mathrm{m}, \mathrm{m}} \cdot \mathcal{R}_{\mathrm{m}, 2}}{\mathcal{R}_{\mathrm{m}, \mathrm{m}}-\mathcal{R}_{\mathrm{m}, 2}}=11.1 \mathrm{MA} \mathrm{Wb}^{-1}$.

\section{(e) Completed equivalent circuit}

The mechanical parameters and magnetomechanical transduction coefficients were determined with Table 4 and the material parameters in Table 3 as well as $E_{2}=71 \mathrm{GPa}$ and $d_{33}=d_{31}=0 \mathrm{~m} \mathrm{~A}^{-1}$ for the aluminum carrier. Figure 31 displays all determined network parameters. It can be deduced that the air path above the coil causes a distinct reduction of the magnetic field strength in the magnetic layer to $12.9 \%$ of the $M M F$ (magnetomotive force). For higher values of $\mu_{r}$ or a larger thickness of the magnetic layer the ratio of the magnetic voltages is approx. $1 / \mu_{r}$. Solenoid conditions, except the transverse coupling, can be achieved either by closing the magnetic path around the turns or eliminating $\mathcal{R}_{\mathrm{m}, 1}$.

\section{(f) Electromechanical transfer function}

The included interaction or feedback of the magnetomechanical transduction causes a further magnetic load. The transfer function $\underline{Z}=\underline{\varphi} / \underline{i}$ can easily be deduced from the electrical impedance. For this reason, the circuit description is simplified in a stepwise manner as shown in Fig. 33. The reluctances $\mathcal{R}_{\mathrm{m}, 1}=\mathcal{R}_{\mathrm{m}, \mathrm{A} \text {, a }}$ and $\mathcal{R}_{\mathrm{m}, 2}$ were transformed in the first step to the electrical side. Then the chained transducers can be combined to one transducer with transduction coefficient $X$. Finally, the bending compliance is transformed to the electrical side, too. If no external moment or deflection is applied, then the transducer can be eliminated. The inductance network represents the magnetic voltage divider and

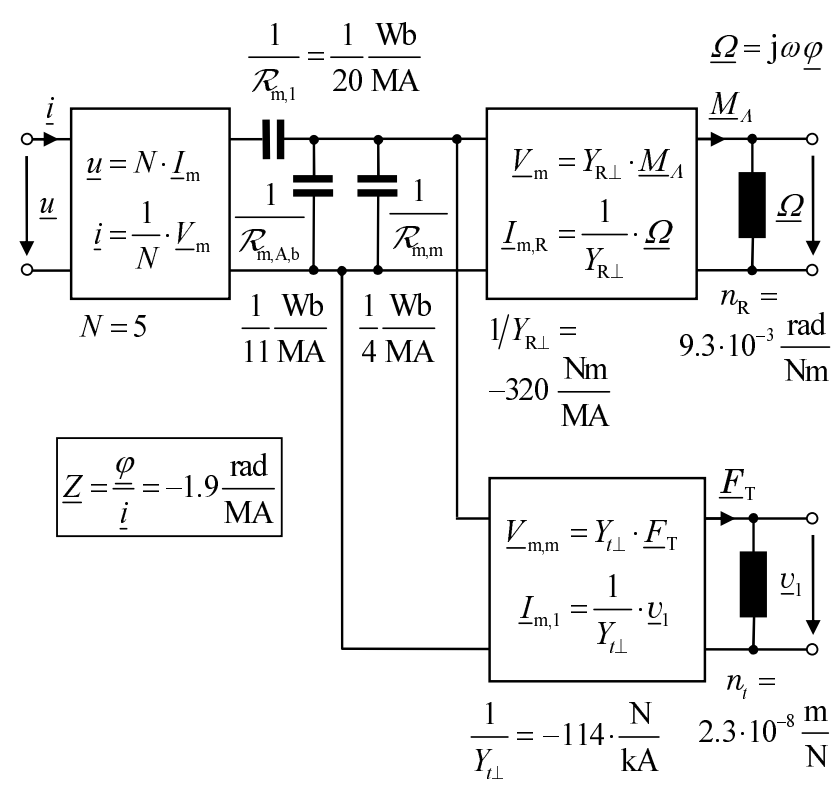

Figure 31. Network parameters of the piezomagnetic unimorph with planar conductor setup from Fig. 26.

the bending compliance. By applying the current divider rule the analytical transfer function follows directly:

$\underline{Z}=\frac{\varphi}{\underline{i}}=n_{\mathrm{R}} \cdot \frac{\underline{M}_{\Lambda}}{\underline{i}}=\frac{n_{\mathrm{R}}}{X} \cdot \frac{L_{1}}{L_{1}+L_{2}+n_{\mathrm{R}} / X^{2}}$.

The value is displayed in Fig. 31 .

\section{(g) Model validity}

The low-frequency model is valid up to a frequency below the first natural frequency of the beam, which follows from the solutions of the Euler-Bernoulli differential equation with

$(\beta l)^{2}=\omega \sqrt{\frac{E \cdot I}{(\varrho \cdot A)}}$,

where $E$ is Young's modulus, $I$ the moment of inertia, $\varrho$ the density and $A$ the cross sectional area. Considering a fixedfree beam as an example, $\left(\beta_{1} l\right)^{2}=3.52$ applies, giving a natural frequency of

$f_{1}=\frac{3.52}{2 \pi} \sqrt{\frac{\overline{E \cdot I}}{\left(h_{1} \varrho_{1}+h_{2} \varrho_{2}\right) w l^{4}}}=372 \mathrm{~Hz}$.

This natural frequency is also found when the high-frequency model of the beam from Fig. 13 is used to simulate the velocity $\hat{v}_{\text {II }}$ of the free beam end. Figure 32 shows the circuit description using the program LTSPICE when the beam is discretized into seven pieces. For the ideal rods, ideal controlled sources were applied. If two $\Delta x / 2$-transducers are connected in series then they can be combined into one transducer with 


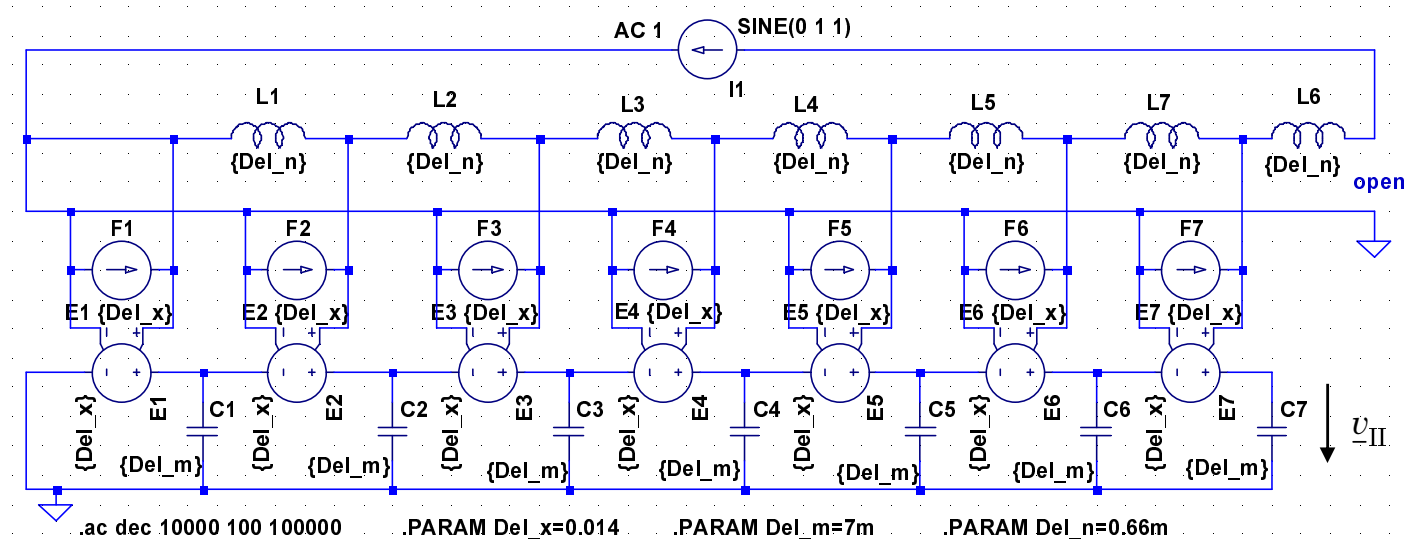

Figure 32. High-frequency LTSpice model of the two-layer beam.

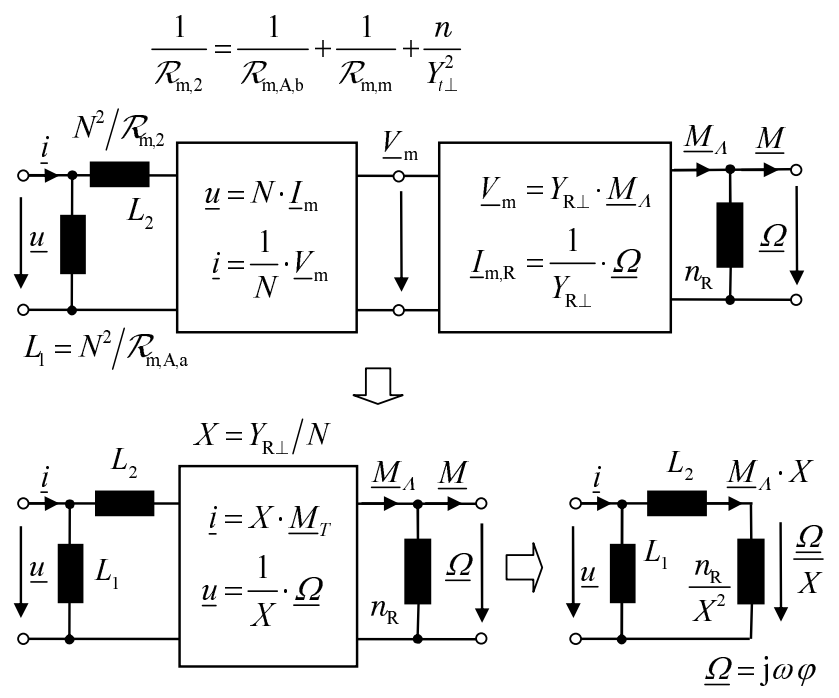

Figure 33. Simplification steps for the setup in Fig. 29 to obtain the electromechanical transfer functions $\varphi / \underline{i}$ and $\underline{\Omega} / \underline{i}$.

$\Delta x=1.4 \mathrm{~cm}$ for the beam of Fig. 26. The free end is stimulated by a moment source with an infinitely large mechanical source impedance. In an electric network, this corresponds to a high-impedance current source. The result of the frequency domain simulation is depicted in Fig. 34. Only six instead of seven natural frequencies are described by this model since the left-side short-circuiting of both, the rotational and the translational domain, is transformed to a short circuit, which is blocking mass $\mathrm{C} 1$. This eliminates $\mathrm{C} 1$.

Despite the small number of finite network elements in the LTSpice model of Fig. 32 and the partial influence of the boundary conditions on the adjacent node, the mechanical network turns out to be a good approximation for the description of the dynamic behavior of the flexure beam including the lower natural frequencies. To improve the results, more network elements (typically nine or more per wavelength) should be used.

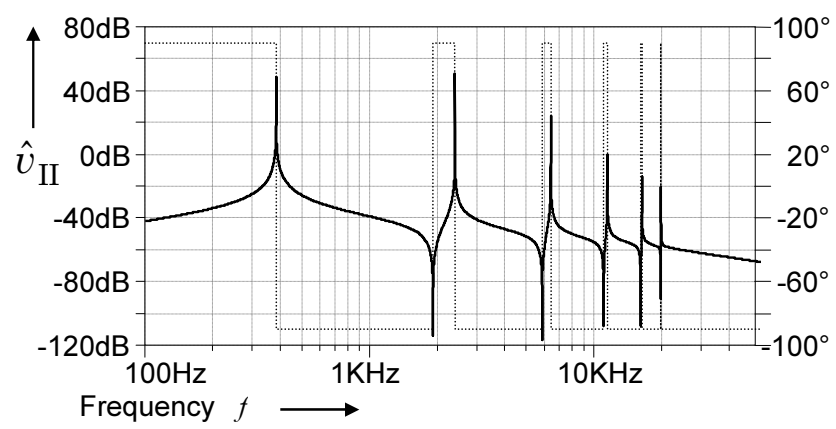

Figure 34. Amplitude and phase spectrum of $\hat{v}_{\text {II }}$ of the left-side fixed bimorph.

\section{Summary}

In this paper a generalized a generalized theory for the mechanical behavior of laminated plate strips or bimorph elements under the influence of strain-inducing physical quantities is presented. These conditions were formulated so that for the description of the bimorph only the uniaxial stress conditions need to be considered. On this basis, it was possible to determine the set of differential equations of the twolayer beam element and to derive a circuit interpretation of the linear case.

The neutral layers caused by an internally induced bending moment and by an externally applied moment are different. The midpoints of the location of the neutral layer at the bimorph ends occurring for an external moment are chosen as points for applying an external longitudinal force. At this location the bimorph length changes when it is actuated. For this reason the equivalent circuit includes three mechanical domains: translational deflection direction, longitudinal direction and bending. The circuit representation of the bimorph element assumes small bending angles as well as small longitudinal forces and neglects quadratic solution terms for the longitudinal direction. It is also assumed that 
Table 4. Piezoelectric (PE) and piezomagnetic (PM) two-layer beam network parameters.

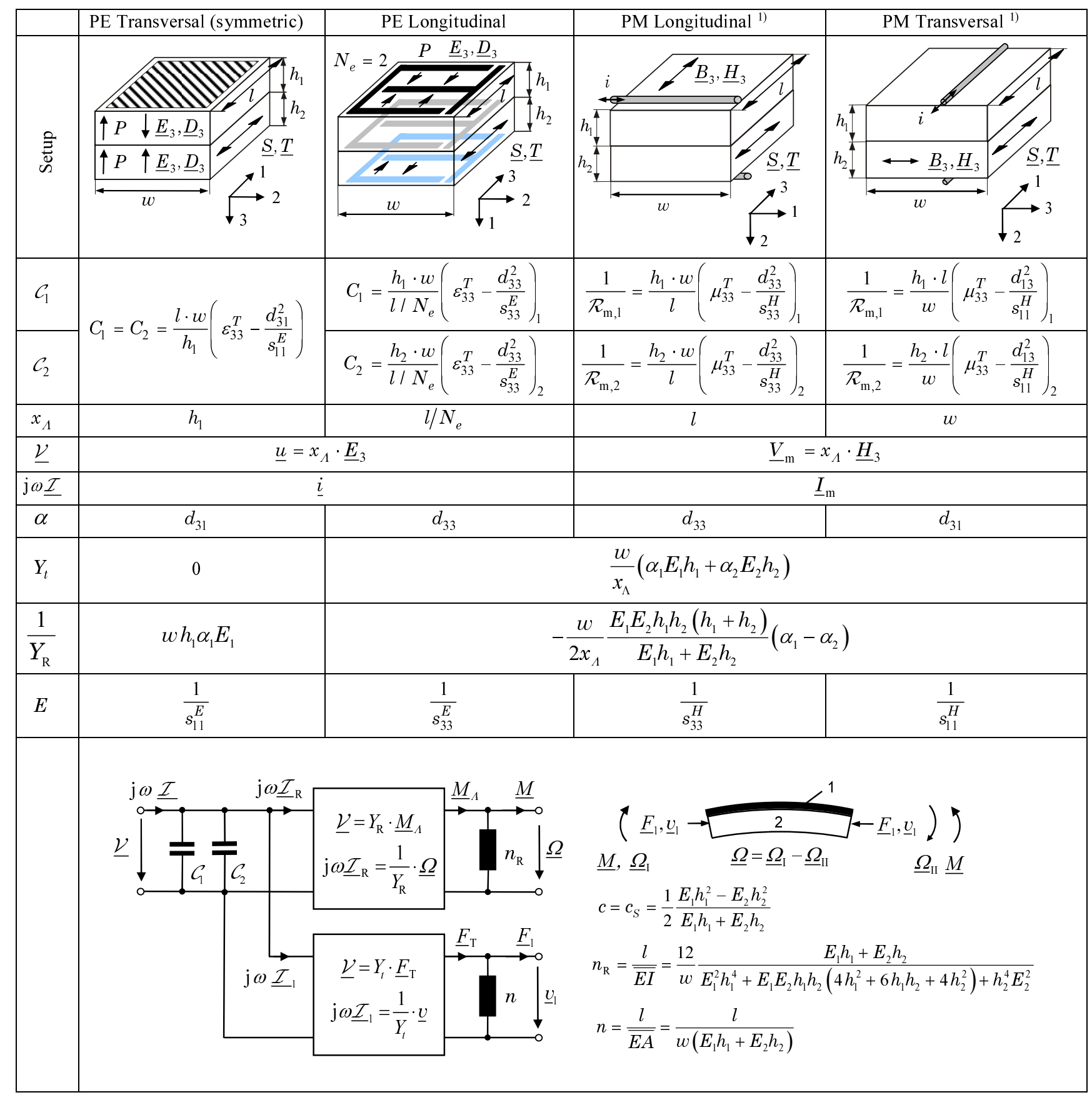

1) The single wire with current i symbolizes the section of a coil

longitudinal compression forces cause only functions of coordinates which fulfill the demand for biuniqueness.

Full bimorph solutions are derived for both quasi-static responses and dynamic responses without pressure load. Quasi-static responses are dynamic responses at frequencies that are well below the first natural frequency of the bimorph, where compliance effects dominate dynamics responses and mass effects are negligible.
If the active layer(s) of the flexure beam behave linearly and reversibly regarding thermodynamics, then an ideal source acts in the interacting domain, too. This additional source and the moment or rotational velocity source define a reversible transducer, which relates the coordinate pairs of interacting domain and mechanical domain to each other. For piezoelectric and piezomagnetic two-layer flexure beams with typical arrangements, reversible multi-port transducer 
models are developed. Piezomagnetic unimorph models are coupled with electromagnetic coil transducers. Except for an asymmetric transversally coupled piezoelectric bimorph, a unified circuit representation is summarized.

The structural description by an equivalent circuit is the key to an efficient analysis, simulation and understanding of the dynamic bimorph behavior. Sophisticated circuit simulators like SPICE offer fast time and frequency domain simulations. When SI units are used, simulation results can be directly read in the related mechanical, magnetic or other domain with their respective units, e.g., Ampère $\rightarrow$ Newton. Because of linearity and reversibility, network elements can be transformed into other physical domains. Subsequent transducers can be combined and eliminated. This way transfer coefficients can be determined easily.

Network methods and finite-element methods can be combined on the one hand in order to determine network parameters. On the other hand completed network models concentrate properties of system parts. They can be integrated in FE models which describe the continua of other system parts. The equivalent circuit of the planar-coil-driven piezomagnetic unimorph, for example, can be integrated into the acoustic FE model of a piezomagnetic loudspeaker to determine electroacoustic function parameters. Such a combined simulation saves time and computational resources. 
Appendix A: Nomenclature

\begin{tabular}{|c|c|c|}
\hline Symbol & Unit & Description \\
\hline $\mathcal{A}$ & & Influence coefficients ratio \\
\hline$B$ & $\mathrm{Vs} \mathrm{m}^{-2}$ & Magnetic flux density \\
\hline$c_{A}$ & $\mathrm{~m}$ & $\begin{array}{l}\text { Distance between neutral layer and } \\
\text { material interface for } \\
\text { actuation }\end{array}$ \\
\hline$c_{S}$ & $\mathrm{~m}$ & $\begin{array}{l}\text { Distance between the neutral layer } \\
\text { and material interface for sensing }\end{array}$ \\
\hline$C_{a}, C_{b}$ & & Deflection function constants \\
\hline$C_{1}, C_{2}$ & $\mathrm{~F}$ & Capacitances of layers 1 and 2 \\
\hline$d_{33}$ & $\begin{array}{l}\mathrm{m} \mathrm{V}^{-1} \\
\mathrm{~m} \mathrm{~A}^{-1}\end{array}$ & $\begin{array}{l}\text { Piezoelectric or piezomagnetic } \\
\text { constant for longitudinal coupling }\end{array}$ \\
\hline$d_{31}$ & $\begin{array}{l}\mathrm{m} \mathrm{V}^{-1} \\
\mathrm{~m} \mathrm{~A}^{-1}\end{array}$ & $\begin{array}{l}\text { Piezoelectric or piezomagnetic } \\
\text { constant for transversal coupling }\end{array}$ \\
\hline$d_{31}^{*}$ & $\begin{array}{l}\mathrm{m} \mathrm{V}^{-1} \\
\mathrm{~m} \mathrm{~A}^{-1}\end{array}$ & $\begin{array}{l}\text { Piezoelectric or piezomagnetic } \\
\text { constant for transversal coupling } \\
\text { and large beam width }\end{array}$ \\
\hline$\underline{D}_{3}$ & As $m^{-2}$ & $\begin{array}{l}\text { Displacement current in active } \\
\text { electrical field direction }\end{array}$ \\
\hline$E=1 / s$ & $\mathrm{~Pa}$ & Young's modulus \\
\hline$\overline{E A}$ & & Averaged extensional stiffness \\
\hline$\overline{E I}$ & & Averaged bending stiffness \\
\hline$\underline{E}$ & $\mathrm{Vm}^{-1}$ & Electric field strength \\
\hline$F_{1}, F_{3}$ & $\mathrm{~N}$ & Forces in directions 1 and 3 \\
\hline$F_{\mathrm{I}}, F_{\mathrm{II}}$ & $\mathrm{N}$ & $\begin{array}{l}\text { Forces at left and right side of a } \\
\text { beam in direction } 3\end{array}$ \\
\hline$F^{*}$ & $\mathrm{~N}$ & $\begin{array}{l}\text { Force at the beam cross section for } \\
\text { large angles }\end{array}$ \\
\hline$h_{1}, h_{2}$ & $\mathrm{~m}$ & Thicknesses of layers 1 and 2 \\
\hline$\underline{H}$ & $\mathrm{~A} \mathrm{~m}^{-1}$ & Magnetic field strength \\
\hline $\bar{i}$ & A & Electrical current \\
\hline$\underline{I}_{\mathrm{m}}$ & $\mathrm{V}$ & Magnetic flux rate \\
\hline $\mathcal{I}$ & & $\begin{array}{l}\text { Generalized time integral of the } \\
\text { flow }\end{array}$ \\
\hline$k$ & & Coupling factor \\
\hline $\mathcal{K}$ & & $\begin{array}{l}\text { Generalized second field quantity in } \\
\text { the interacting domain }\end{array}$ \\
\hline$l$ & $\mathrm{~m}$ & Beam length \\
\hline$L$ & $\mathrm{H}$ & Inductance \\
\hline$\underline{M}$ & $\mathrm{Nm}$ & Moment \\
\hline$\underline{\bar{M}}_{\Lambda}$ & $\mathrm{Nm}$ & Source moment \\
\hline$\underline{M}_{\mathrm{I}}, \underline{M}_{\mathrm{II}}$ & $\mathrm{Nm}$ & $\begin{array}{l}\text { Moment at left and right side of a } \\
\text { beam }\end{array}$ \\
\hline$n$ & $\mathrm{~m} \mathrm{~N}^{-1}$ & Compliance \\
\hline$n_{0}$ & $\mathrm{~m} \mathrm{~N}^{-1}$ & Reference compliance \\
\hline$n_{F_{1}}$ & $\operatorname{rad~Nm}{ }^{-1}$ & Rotational compliance related to $F_{1}$ \\
\hline$n_{\mathrm{R}}$ & $\operatorname{rad} \mathrm{Nm}^{-1}$ & Rotational compliance \\
\hline$N$ & & $\begin{array}{l}\text { Number of turns; } \\
\text { number of discrete elements }\end{array}$ \\
\hline$N_{\mathrm{e}}$ & & $\begin{array}{l}\text { Number of interdigital electrodes } \\
\text { minus one }\end{array}$ \\
\hline$p$ & $\mathrm{~Pa}$ & Pressure \\
\hline$Q$ & As & Electric charge \\
\hline$\overline{\mathcal{R}}_{\mathrm{m}}$ & $\mathrm{A} \mathrm{Wb}^{-1}$ & Magnetic reluctance \\
\hline$s$ & $\mathrm{~m}^{2} \mathrm{~N}^{-1}$ & Elasticity constant \\
\hline$s_{i j}^{E}$ & $\mathrm{~m}^{2} \mathrm{~N}^{-1}$ & Elasticity constant for $E=0$ \\
\hline
\end{tabular}

\begin{tabular}{|c|c|c|}
\hline Symbol & Unit & Description \\
\hline$s_{i j}^{E *}$ & $\mathrm{~m}^{2} \mathrm{~N}^{-1}$ & $\begin{array}{l}\text { Elasticity constant for } E=0 \text { and } \\
\text { large beam width }\end{array}$ \\
\hline$s_{i j}^{H}$ & $\mathrm{~m}^{2} \mathrm{~N}^{-1}$ & Elasticity constant for $H=0$ \\
\hline$\underline{S}$ & & Strain \\
\hline$\underline{S}_{0}$ & & Strain at $c_{S}$ and $x_{3}=0$ \\
\hline$t$ & $\mathrm{~s}$ & Time \\
\hline$\underline{T}$ & $\mathrm{Nm}^{-2}$ & Stress \\
\hline$\underline{T}_{B}$ & $\mathrm{~N} \mathrm{~m}^{-2}$ & $\begin{array}{l}\text { Mean-free part of the stress } \\
\text { distribution }\end{array}$ \\
\hline$\underline{u}$ & $\mathrm{~V}$ & Voltage \\
\hline $\bar{v}_{1}, \underline{v}_{3}$ & $\mathrm{~m} \mathrm{~s}^{-1}$ & Velocity in directions 1 and 3 \\
\hline$\underline{v}_{\mathrm{I}}, \underline{v}_{\mathrm{II}}$ & $\mathrm{m} \mathrm{s}^{-1}$ & $\begin{array}{l}\text { Velocity at left and right side of a } \\
\text { beam in direction } 3\end{array}$ \\
\hline$\underline{V}_{\mathrm{m}}$ & A & $\begin{array}{l}\text { Magnetomotive force }(M M F) \text { or } \\
\text { magnetic voltage }\end{array}$ \\
\hline$\underline{\mathcal{V}}$ & & $\begin{array}{l}\text { Generalized influence field } \\
\text { strength }\end{array}$ \\
\hline$w$ & $\mathrm{~m}$ & Beam width \\
\hline$x_{1}, x_{2}, x_{3}$ & $\mathrm{~m}$ & Mechanical coordinate axes \\
\hline$X$ & & Interacting domain \\
\hline$X_{t \perp}$ & & $\begin{array}{l}\text { Translational electromechanical } \\
\text { transduction coefficient for } \\
\text { transverse coupling }\end{array}$ \\
\hline$X_{\mathrm{R} \perp}$ & & $\begin{array}{l}\text { Rotational electromechanical } \\
\text { transduction coefficient for } \\
\text { transverse coupling }\end{array}$ \\
\hline$Y_{t}$ & $\begin{array}{l}\mathrm{VN}^{-1} \\
\mathrm{AN}^{-1}\end{array}$ & $\begin{array}{l}\text { Translational transduction } \\
\text { coefficient }\end{array}$ \\
\hline$Y_{\mathrm{R}}$ & $\begin{array}{l}\mathrm{V}(\mathrm{Nm})^{-1} \\
\mathrm{~A}(\mathrm{Nm})^{-1}\end{array}$ & Rotational transduction coefficient \\
\hline$\underline{Z}$ & $\operatorname{rad} A^{-1}$ & Electromechanical transfer function \\
\hline $\bar{\alpha}_{1}, \alpha_{2}$ & & $\begin{array}{l}\text { Generalized influence coefficient of } \\
\text { layers } 1 \text { and } 2\end{array}$ \\
\hline$\beta_{1}, \beta_{2}$ & & $\begin{array}{l}\text { Generalized material coefficient of } \\
\text { layers } 1 \text { and } 2\end{array}$ \\
\hline$\varepsilon$ & $\mathrm{F} \mathrm{m}^{-1}$ & Permittivity \\
\hline$\varepsilon_{m n}^{S}$ & $\mathrm{~F} \mathrm{~m}^{-1}$ & Permittivity for $S=0$ \\
\hline$\varepsilon_{m n}^{T}$ & $\mathrm{Fm}^{-1}$ & Permittivity for $T=0$ \\
\hline$\varepsilon_{m n}^{T *}$ & $\mathrm{~F} \mathrm{~m}^{-1}$ & $\begin{array}{l}\text { Permittivity for } T=0 \text { and large } \\
\text { beam width }\end{array}$ \\
\hline$\zeta$ & & Layer thickness ratio \\
\hline$\vartheta$ & $\mathrm{K}$ & Temperature \\
\hline$\Lambda$ & & Generalized influence quantity \\
\hline$\mu_{\mathrm{r}}$ & & Relative permeability \\
\hline$\mu_{m n}^{T}$ & $\operatorname{Vs}(\mathrm{Am})^{-1}$ & Permeability for $T=0$ \\
\hline$\mu_{m n}^{T *}$ & $\mathrm{Vs}(\mathrm{Am})^{-1}$ & $\begin{array}{l}\text { Permeability for } T=0 \text { and large } \\
\text { beam width }\end{array}$ \\
\hline$v$ & & Poisson's ratio \\
\hline$\underline{\varphi}$ & $\mathrm{rad}$ & Angle \\
\hline$\underline{\Phi}$ & $\mathrm{Wb}$ & Magnetic flux \\
\hline$\xi_{1}, \xi_{3}$ & $\mathrm{~m}$ & Deflections in directions 1 and 3 \\
\hline$\xi_{1}^{*}, \xi_{3}^{*}$ & $\mathrm{~m}$ & Transformed deflections $\xi_{1}, \xi_{3}$ \\
\hline$\chi$ & & Young's modulus ratio \\
\hline$\Psi$ & $\%$ & Humidity \\
\hline$\underline{\Omega}$ & $\operatorname{rad~s}^{-1}$ & Rotational velocity \\
\hline $\bar{\omega}$ & $\operatorname{rads}^{-1}$ & Circular frequency \\
\hline$\underline{\Omega}_{\mathrm{I}}, \underline{\Omega}_{\mathrm{II}}$ & $\operatorname{rads}^{-1}$ & $\begin{array}{l}\text { Rotational velocity at left and right } \\
\text { side of a beam }\end{array}$ \\
\hline
\end{tabular}


Acknowledgements. This Work was supported in part by the Deutsche Forschungsgemeinschaft (German Research Foundation) within the Collaborative Research Center (SFB) 639 "Textilereinforced composite components for function-integrating multimaterial design in complex lightweight applications", subproject D3.

The authors thank Günther Pfeifer from the Technische Universität Dresden for his advice concerning Combined Simulation. We also thank Alison B. Flatau from the University of Maryland and Matthias Plötner from the Technische Universität Dresden, who gave insightful comments and suggestions. Axel Renner from the Technische Universität Dresden and Jin-Hyeong Yoo from the University of Maryland helped solving ANSYS-problems, which is gratefully acknowledged, too.

Edited by: A. Schütze

Reviewed by: three anonymous referees

\section{References}

Gerlach, G. and Dötzel, W.: Introduction to Microsystem Technology, John Wiley \& Sons, 2008.

Gerlach, G. and Lenk, A.: Biegetheorie des geschichteten Plattenstreifens mit inneren Dehnungsquellen, Discussion paper, Tech. rep., Technische Universität Dresden, Sektion Informationstechnik, 1985.

Guerrero, V. H. and Wetherhold, R. C.: Magnetostrictive bending of cantilever beams and plates, J. Appl. Phys., 94, 6659-6666, 2003.

Kašpar, F.: Thermobimetalle in der Elektrotechnik, Verlag Technik Berlin, 1960.

Lenk, A. and Irrgang, B.: Elektromechanische Systeme, Band 2: Systeme mit verteilten Parametern, Verlag Technik Berlin, 1977.

Lenk, A., Ballas, R., Werthschützky, R., and Pfeifer, G.: Electromechanical Systems in Microtechnology and Mechatronics, Springer, 2011.
Marschner, U., Starke, E., Pfeifer, G., Fischer, W.-J., and Flatau, A. B.: Electromagnetic network models of planar coils on a thin or thick magnetic layer, IEEE Transactions on Magnetics, 46, 2365-2368, 2010.

Marschner, U., Datta, S., Starke, E., Fischer, W.-J., and Flatau, A.: Equivalent Circuit of a Piezomagnetic Unimorph Incorporating Single Crystal Galfenol, accepted, IEEE Transactions on Magnetics, doi:10.1109/TMAG.2014.2323362, 2014.

Mudivarthi, C., Datta, S., Atulasimha, J., and Flatau, A. B.: A bidirectionally coupled magnetoelastic model and its validation using a Galfenol unimorph sensor, Smart Materials and Structures, 17, 035005, available at: http://stacks.iop.org/0964-1726/17/i=3/ $\mathrm{a}=035005$ (last access: 21 August 2014), 2008.

Pflüger, A.: Stabilitätsprobleme der Elastostatik, Springer, Berlin, Heidelberg, New York, 3 Edn., 1975.

Roshen, W. and Turcotte, D.: Planar inductors on magnetic substrates, IEEE Transactions on Magnetics, 24, 3213-3216, 1988.

Schroth, G.: Die näherungsweise Darstellung eines Biegestabes durch konzentrierte Schaltelemente, Hochfrequenztechnik und Elektroakustik, p. 68, 1968.

Starke, E.: Kombinierte Simulation - eine weitere Methode zur Optimierung elektromechanischer Systeme, Ph.D. thesis, Technische Universität Dresden, Fakultät Elektrotechnik und Informationstechnik, 2009.

Starke, E., Marschner, U., Pfeifer, G., Fischer, W.-J., and Flatau, A. B.: Combining network models and FE-models for the simulation of electromechanical systems, in: SPIE, edited by: GhasemiNejhad, M. N., Vol. 7977, pp. 79771Y-1-8, 2011.

Stoney, G. G.: The tension of metallic films deposited by electrolysis, P. Roy. Soc. London A, 82, 172-175, available at: http://rspa. royalsocietypublishing.org/content/82/553/172.short, 1909. 